hep-th/0506224

\title{
Uplifting the Iwasawa
}

\author{
Anne Franzen $^{(i)(i i) 1}$, Payal Kaura ${ }^{(i i) 2}$, Aalok Misra ${ }^{(i i),(i i i) 3}$ and \\ Rajyavardhan Ray ${ }^{(i i) 4}$ \\ (i) Department of Physics, Rheinisch-Westfälische Technische \\ Hochschule Aachen, 52056 Aachen, Germany \\ (ii) Department of Physics, Indian Istitute of Technology Roorkee, \\ Roorkee 247 667, India \\ (iii) Jefferson Physical Laboratory, Harvard University, Cambridge, \\ MA 02138, USA
}

\begin{abstract}
The Iwasawa manifold is uplifted to seven-folds of either $G_{2}$ holonomy or $S U(3)$ structure, explicit new metrics for the same having been constructed in this work. We uplift the Iwasawa manifold to a $G_{2}$ manifold through "size" deformation (of the Iwasawa metric), via Hitchin's Flow equations, showing also the impossibility of the uplift for "shape" and "size" deformations (of the Iwasawa metric). Using results of [1], we also uplift the Iwasawa manifold to a 7-fold with $S U(3)$ structure through "size" and "shape" deformations via generalisation of Hitchin's Flow equations. For seven-folds with $S U(3)$-structure, the result could be interpreted as $M 5$-branes wrapping two-cycles embedded in the seven-fold (as in [1]) - a warped product of either a special hermitian six-fold or a balanced six-fold with the unit interval. There can be no uplift to seven-folds of $S U(3)$ structure involving non-trivial "size" and "shape" deformations (of the Iwasawa metric) retaining the "standard complex structure" - the uplift generically makes one move in the space of almost complex structures such that one is neither at the standard complex structure point nor at the "edge". Using the results of [2], we show that given two "shape deformation" functions, and the dilaton, one can construct a Riemann surface obtained via Weierstraß representation for the conformal immersion of a surface in $\mathbf{R}^{l}$, for a suitable $l$, with the condition of having conformal immersion being a quadric in $\mathbf{C} \mathbf{P}^{l-1}$.
\end{abstract}

\footnotetext{
${ }^{1}$ e-mail:anne-franzen@web.de

${ }^{2}$ e-mail: pa123dph@iitr.ernet.in

${ }^{3}$ e-mail: aalokfph@iitr.ernet.in

${ }^{4}$ e-mail: riitrpph@iitr.ernet.in
} 


\section{Introduction}

Unlike the standard model, which contains many free parameters, it is expected that string theory, should be able to do a much better job, as regards explaining things like quark and lepton masses and the gauge hierarchy, etc, from the vacuum expectation values (vev's) of the moduli fields. In addition to giving vevs to the moduli $([3,4])$, string compactifications in the presence of fluxes, can also give a positive cosmological constant [5], making the study even more phenomenologically interesting. The inclusion of fluxes necessarily requires the introduction of warp factors in the metric $[8,4,9,10,11]$, which can be argued to provide a mechanism for generating the large hierarchy of scales - the (not-so) recent TeV-scale quantum gravity [12] proposal being particularly significant. The study of fluxes is also of much importance from the point of view of open/closed (topological) string dualities [6, 7]. Thus, turning on fluxes is of great importance for establishing connection between string theory and the observable universe.

Turning on $N S-N S$ fluxes in, e.g., Heterotic theory, the internal manifold cannot be Kähler anymore implying that one then has torsion. As shown in $[8,13]$, Iwasawa manifold, an example of a "half-flat" manifold (see also [15] and references therein) satisfies the conditions for $\mathcal{N}=1$ supersymmetry in the presence of $N S-N S$ fluxes. Based on [13,1], we study the explicit uplifts of the Iwasawa metric at the "standard complex structure" point of the moduli space of almost complex structures, to seven-folds with either $G_{2}$ holonomy or $S U(3)$ structure.

The plan of the paper is as follows. In Section 2, we give a brief review of six-folds with $S U(3)$ structure, the relevant torsion classes and the Iwasawa manifold. In Section 3, we discuss the uplift of the Iwasawa manifold to seven-folds of $G_{2}$ holonomy using Hitchin's flow equations using "size" deformations and we show the impossibility of the uplift using "size" and "shape" deformations of the Iwasawa via Hitchin's flow equations. We then give a brief review of sevenfolds with $S U(3)$ structure and the relevant torsion classes and then discuss the uplift of the Iwasawa (at the "standard complex structure" point) to seven-folds with $S U(3)$ structure using "size" and "shape" deformations via generalizations of the Hitchin's flow equations. Section 4 has the interpretation of the non-vanishing Bianchi identity for $M$-theory compactified on sevenfolds with $S U(3)$ structure, in the presence of $G$-flux sourced by $M 5$-branes wrapping two-cycles embedded in the seven-fold (analagous to [1]) and the generic movement in the moduli space of almost complex structures on the Iwasawa by "size" and "shape" deformations of the Iwasawa metric. Using the results of [2], we show that one can associate a Riemann surface with a pair of "shape deformation" functions and the dilaton, via the Weierstraß representation for the conformal immersion of a surface in $\mathbf{R}^{l}$ for a suitable $l$. Section 5 has the conclusion. There are two appendices. 


\section{Six-Folds with $S U(3)$ Structure}

In this section, we give a brief review of six-folds with $S U(3)$ structure, relevant to, e.g., Heterotic theory compactifications in the presence of $N S-N S$ flux, and a short review of the "half-flat" Iwasawa manifold.

\subsection{Brief review of torsion classes}

As per the work of [8], the requirements for $\mathcal{N}=1$ supersymmetric compactification of heterotic string theory are:

(a) The internal 6-dim manifold has to be complex. That means that the Nijenhuis tensor $N_{m n p}$ has to vanish.

(b) Up to a constant factor, there is exactly one holomorphic (3,0)-form $\omega$ whose norm is related to the complex structure $J$ by $\star d \star J=i(\bar{\partial}-\partial) \log \|\omega\|$.

(c) The Yang Mills background field strength must be a (1,1)-form and must satisfy $\operatorname{tr} F \wedge F=$ $\operatorname{tr} \tilde{R} \wedge \tilde{R}-i \partial \bar{\partial} J$ as well as $F_{m n} J^{m n}=0$ (the Donaldson-Uhlenbeck-Yau condition), $\tilde{R}$ being the modified curvature two-form in the presence of torsion.

(d) The Warp factor is given by $\triangle(y)=\phi(y)+$ const ; the dilaton by $\phi(y)=\frac{1}{8} \log \|\omega\|+$ const.

(e) The background 3-form $H$ is determined in terms of $J$ by $H=\frac{i}{2}(\bar{\partial}-\partial) J$.

It is possible to reformulate these conditions in terms of torsional constraints [13, 14]. NS-NS flux $\neq 0$ requires $\tilde{Y}$ to be a manifold with $S U(3)$ structure but not $S U(3)$ holonomy. $S U(3)$ structure implies: $\exists \mathrm{J}, \Omega$ such that $d J \neq 0 ; d \Omega \neq 0$ which means that the Manifold is not Kähler and then can also not be CY. $S U(3)$-structure can now be determined in terms of torsion classes.

The difference between any two metric-compatible connections is a tensor, known as the contorsion $\kappa_{m n p}$ defined via:

$$
\nabla_{m}^{(T)} \eta=\nabla_{m} \eta-\frac{1}{4} \kappa_{m n p} \Gamma^{n p} \eta=0
$$

$\eta$ being the globally defined spinor that is covariantly constant w.r.t. the connection modified by the the three-form flux $H$. The contorsion can be related to torsion $T_{m n p}$ through

$$
T_{m n p}=\frac{1}{2}\left(\kappa_{m n p}-\kappa_{n m p}\right) .
$$

The torsion classes can be defined in terms of J, $\Omega$, dJ, $d \Omega$ and the contraction operator $\_\mid$: $\Lambda^{k} T^{\star} \otimes \Lambda^{n} T^{\star} \rightarrow \Lambda^{n-k} T^{\star}$ where $J$ is given by:

$$
J=e^{1} \wedge e^{2}+e^{3} \wedge e^{4}+e^{5} \wedge e^{6}
$$

and the $(3,0)$-form $\Omega$ is given by

$$
\Omega=\left(e^{1}+i e^{2}\right) \wedge\left(e^{3}+i e^{4}\right) \wedge\left(e^{5}+i e^{6}\right) .
$$


The basis of $(1,0)$-forms is given by

$$
e^{i}+i J \cdot e^{i} \in \Lambda^{(1,0)}
$$

where $J \cdot e^{a}=J^{a}{ }_{b} e^{b}$ and consequently $J \cdot J=-1$.

The torsion classes are defined in the following way: $W_{1} \leftrightarrow[d J]^{(3,0)}$, given by real numbers $W_{1}=W_{1}^{+}+W_{1}^{-}$with $d \Omega_{+} \wedge J=\Omega_{+} \wedge d J=W_{1}^{+} J \wedge J \wedge J$ and $d \Omega_{-} \wedge J=\Omega_{-} \wedge d J=W_{1}^{-} J \wedge J \wedge J$; similarly for $W_{2} \leftrightarrow[d \Omega]_{0}^{(2,2)}:\left(d \Omega_{+}\right)^{(2,2)}=W_{1}^{+} J \wedge J+W_{2}^{+} \wedge J$ and $\left(d \Omega_{-}\right)^{(2,2)}=W_{1}^{-} J \wedge J+W_{2}^{-} \wedge J$; $W_{3} \leftrightarrow[d J]_{0}^{(2,1)}$ is defined as $W_{3}=d J^{(2,1)}-\left[J \wedge W_{4}\right]^{(2,1)} ; W_{4} \leftrightarrow J \wedge d J: W_{4}=\frac{1}{2} J_{-} \mid d J ;$

$W_{5} \leftrightarrow[d \Omega]_{0}^{(3,1)}: W_{5}=\frac{1}{2} \Omega_{+-} \mid d \Omega_{+}$(the subscript 0 indicative of the primitivity of the respective forms). Depending on the classes of torsion one can obtain different types of manifolds:

- (complex) special-hermitian manifolds with $W_{1}=W_{2}=W_{4}=W_{5}=0$ which means that $\tau \in W_{3}$

- (complex) Kähler manifolds with $W_{1}=W_{2}=W_{3}=W_{4}=0$ which means $\tau \in W_{5}$;

- (complex) balanced Manifolds with $W_{1}=W_{2}=W_{4}=0$ which means $\tau \in W_{3} \oplus W_{5}$;

- (complex) Calabi-Yau manifolds with $W_{1}=W_{2}=W_{3}=W_{4}=W_{5}=0$ which means $\tau=0$.

- half-flat manifolds (may or may not be complex) with $W_{1}=W_{2}=0$ whch means $\tau \in$ $W_{3} \oplus W_{4} \oplus W_{5}$.

\subsection{Heterotic string on the Iwasawa manifold}

The torsional constraints are: $\tau \in W_{3} \oplus W_{4} \oplus W_{5} ; 2 W_{4}+W_{5}=0$ and $W_{4,5}$ real, exact. We consider now manifolds fullfilling the torsional constraints and satisfying supersymmetry requirements. From this we obtain, that we need special-hermitian manifolds for which the torsion is $\tau \in W_{3}$; they are complex and half-flat. Since $W_{4}=W_{5}=0$ it follows that the dilaton is constant. We consider nilmanifolds (6-dim) which are special-hermitian manifolds which are constructed from simply-connected nilpotent Lie group $\mathrm{G}$ by quotienting with discrete subgroup $\Gamma$ of $\mathrm{G}$ for which $G \backslash \Gamma$ is compact.

There are 34 classes of such manifold and they do not admit a Kähler metric. 18 of these admit complex structure. An example is the Iwasawa manifold. For any of 18 classes one can choose complex structure, compatible with metric and $W_{4}=0$. Iwasawa manifold is a nilmanifold obtained as the compact quotient space $M=\Gamma \backslash G$, where $G$ the complex Heisenberg group is given by a set of matrices under multiplication

$$
G=\left\{\left(\begin{array}{ccc}
1 & z & u \\
0 & 1 & v \\
0 & 0 & 1
\end{array}\right): u, v, z \in C\right\} .
$$


The discrete subgroup $\Gamma$ is defined by restricting $u, v, z$ to Gaussian integers:

$$
\begin{aligned}
& v \rightarrow v+m \\
& z \rightarrow z+m \\
& u \rightarrow u+p+n v
\end{aligned}
$$

where $m, n, p \in Z \oplus i Z$. To find an Iwasawa manifold solution of torsional constraints the parameters have to be choosen such that the torsion lies in $\tau \in W_{3}$ with $2 W_{4}+W_{5}=0$ and $W_{4}=W_{5}=0$. It turns out that the moduli space of complex structures has two disconnected components referred as the standard complex structure $J_{0}$ and 'edge'. It can be shown that in both components $W_{4}=W_{5}=0$.

The Standard complex stucture is given by: $J_{0}=e^{1} \wedge e^{2}+e^{3} \wedge e^{4}+e^{5} \wedge e^{6}$ with the (1,0)-forms are given by

$$
\alpha=e^{1}+i e^{2}, \quad \beta=e^{3}+i e^{4}, \quad \gamma=e^{5}-i e^{6} .
$$

Complex coordinates $(z, v, u)$ can be introduced

$$
\begin{aligned}
\alpha & =d z \\
\beta & =d v \\
\gamma & =i(d u-z d v) .
\end{aligned}
$$

These are holomorphic left-invariant $(1,0)$-forms with respect to the standard complex structure.

The two-form in the standard complex structure limit, is given by

$$
J_{0}=\frac{i}{2}[d z \wedge d \bar{z}+d v \wedge d \hat{v}+(d u-z d v) \wedge(d \hat{u}-\bar{z} d \bar{v})]
$$

thereby implying that the metric is $d s^{2}=|d z|^{2}+|d v|^{2}+|d u-z d v|^{2}$. The Iwasawa manifold can thus be viewed as $T^{2}$ fibration over a $T^{2} \times T^{2}$ base. The Euler characteristic for the same is hence zero. The 3-form is given by $\Omega=\alpha \wedge \beta \wedge \gamma=i d z \wedge d v \wedge d u$.

\section{Uplift}

$\mathcal{N}=1$ theories in four dimensions starting from M-theory in the presence of $\mathrm{G}$ ( $\equiv 4$-form) fluxes require 7 -folds with either $G_{2}$ holonomy or $S U(3)$ structure. We first discuss the uplift of the Iwasawa to seven-folds with $G_{2}$ holonomy. 


\subsection{Hitchin's construction of 7-folds with $G_{2}$ Holonomy from 6-folds that are half-flat}

From a six dimensional manifold $M$ with $S U(3)$ structure $(M, J, \Omega)$ one can construct a 7 -dim manifold as a warped product

$$
X_{7}=M \times I ; I \subset \mathbf{R} .
$$

A $G_{2}$-Manifold is defined by: $\phi=J \wedge d t+\Omega_{+}$where the calibration $\phi$ is closed and coclosed and $\left(J, \Omega_{+}, \Omega_{-}\right)$are $t$-dependent. This implies:

$$
\begin{aligned}
& d \phi=\left(\hat{d} J-\frac{\partial \Omega_{+}}{\partial t}\right) \wedge d t+\hat{d} \Omega_{+}=0 \\
& d \star \phi=\left(\hat{d} \Omega_{-}-J \wedge \frac{\partial J}{\partial t}\right) \wedge d t+J \wedge \hat{d} J=0 .
\end{aligned}
$$

Through this the forms have now been promoted to seven dimensions. $M$ is half-flat which means that its $S U(3)$ structure is such that:

$$
\hat{d} \Omega_{+}=J \wedge \hat{d} J=0 .
$$

The conditions (1) for the seven-manifold $X_{7}$ to have $G_{2}$ holonomy, i.e. $d \phi=d \star \phi=0$, yield:

$$
\begin{aligned}
\hat{d} J & =\frac{\partial \Omega_{+}}{\partial t} \\
\hat{d} \Omega_{-} & =-J \wedge \frac{\partial J}{\partial t}
\end{aligned}
$$

called Hitchin's Flow equations.

\subsection{Uplift of the Iwasawa manifold to $G_{2}$-holonomy manifold via Hitchin's Flow equation}

In this subsection, we discuss the uplift of the Iwasawa to a seven-fold of $G_{2}$-holonomy using "size" (implying $t$-dependent) deformation of the Iwasawa metric. We later show that "size" alongwith nontrivial "shape" (implying $z, \bar{z} ; v, \bar{v}$-dependent) deformations of the Iwasawa metric can not be used to uplift to a $G_{2}$-holonomy manifold.

We use the following ansatz for $J$ with "size" deformation:

$$
\begin{aligned}
J & =e^{a(t)} e^{12}+e^{b(t)} e^{34}+e^{c(t)} e^{56} \\
\Rightarrow \Omega & =e^{\frac{1}{2}(a(t)+b(t)+c(t))}\left(e^{1}+i e^{2}\right) \wedge\left(e^{3}+i e^{4}\right) \wedge\left(e^{5}+i e^{6}\right)
\end{aligned}
$$

We now use the following two-step algebra:

$$
\hat{d} e^{1}=\hat{d} e^{2}=\hat{d} e^{3}=\hat{d} e^{4}=0 ; \quad \hat{d} e^{5}=e^{14}+e^{23} ; \quad \hat{d} e^{6}=e^{13}+e^{42} ;
$$


with the representations:

$$
\begin{aligned}
& e^{1}+i e^{2}=d z ; \quad e^{3}+i e^{4}=d v ; \quad e^{5}-i e^{6}=i(d u-z d v) . \\
& \Rightarrow \hat{d} J= e^{c(t)}\left(e^{146}+e^{236}-e^{513}-e^{542}\right)
\end{aligned}
$$

The condition $\hat{d} \Omega_{+}=J \wedge \hat{d} J=0$ is identically fullfilled. We solve $\hat{d} \Omega_{-}=-J \wedge \frac{\partial J}{\partial t}$ :

$$
\begin{aligned}
\Rightarrow \dot{a}=\dot{b} & =-\dot{c} ; \\
\frac{d}{d t}\left(e^{a+b}\right) & =-4 e^{\frac{a+b+c}{2}}
\end{aligned}
$$

These are consistent with $\hat{d} J=\frac{\partial \Omega_{+}}{\partial t}$. For the metric we then obtain a one-parameter $(\lambda)$ family of singular $G_{2}$-metrics:

$$
d s_{7}^{2}=d t^{2}+(1+\lambda t)^{\frac{2}{3}}|d z|^{2}+(1+\lambda t)^{\frac{2}{3}}|d v|^{2}+(1+\lambda t)^{-\frac{2}{3}}|d u-z d v|^{2} .
$$

By specifying suitable boundary conditions at the end-points of the interval, one could perhaps use such manifolds in (heterotic-) $M$-theory (like) compactifications.

It is not possible to uplift the Iwasawa to $G_{2}$-manifold via both "size" and (non-trivial) "shape" deformations of the Iwasawa metric. We show the same now. Using e.g. the following ansatz:

$$
\begin{aligned}
& J=e^{a(t)} e^{12}+A(z, \bar{z}, v, \bar{v}) e^{b(t)} e^{34}+B(z, \bar{z}, v, \bar{v}) e^{c(t)} e^{56} \\
& \Omega=e^{\frac{a+b+c}{2}}\left(e^{A_{1}} e^{1}+i e^{A_{2}} e^{2}\right) \wedge\left(e^{A_{3}} e^{3}+i e^{A_{4}} e^{4}\right) \wedge\left(e^{A_{5}} e^{5}+i e^{A_{6}} e^{6}\right),
\end{aligned}
$$

with "shape" and "size" deformation we will obtain from the Hitchin's Flow equations, the conditions for halfflatness and the additional condition $\Sigma A_{i}=A+B$. From the halfflatness condition $\hat{d} \Omega_{+}=0$ we obtain:

$$
\begin{aligned}
& A_{135 z}=A_{135 \bar{z}} \\
& A_{135 v}=A_{135 \bar{v}} \\
& A_{146 z}=A_{146 \bar{z}} \\
& A_{146 v}=-A_{146 \bar{v}} \\
& A_{236 z}=-A_{236 \bar{z}} \\
& A_{236 v}=A_{236 \bar{v}} \\
& A_{245 z}=-A_{245 \bar{z}} \\
& A_{245 v}=-A_{245 \bar{v}},
\end{aligned}
$$

where $A_{i j k_{z} \text { or } \bar{z}} \equiv \frac{\partial\left(A_{i}+a_{j}+A_{l}\right)}{\partial z(\text { or } \bar{z})}$. From the halfflatness condition $J \wedge \hat{d} J=0$ we obtain:

$$
\begin{aligned}
& A_{z}=B_{z} \\
& A_{\bar{z}}=B_{\bar{z}} .
\end{aligned}
$$


Considering the Hitchin's Flow equation $\frac{\partial \Omega_{+}}{\partial t}=\hat{d} J$ it follows:

$$
\begin{aligned}
& -\frac{\dot{a}+\dot{b}+\dot{c}}{2} e^{\frac{a+b+c}{2}} e^{A_{i j k}}=e^{c} e^{B} \\
& (i j k=135 ; 146 ; 236 ; 245) \\
& \Rightarrow A_{135}=A_{146}=A_{236}=A_{245} .
\end{aligned}
$$

The above equations give us

$$
A_{135}=A_{146}=A_{236}=A_{245}=\text { const }
$$

From the other Hitchin's Flow equation $\hat{\Omega_{-}}=-J \wedge \frac{\partial J}{\partial t}$ we obtain:

$$
\begin{aligned}
& \dot{a}=\dot{b}=-\dot{c} \\
& -e^{A} e^{a+b}(\dot{a}+\dot{b})=e^{A_{136}}+e^{A_{145}}+e^{A_{235}}+e^{A_{246}} \\
& A_{136 z}=A_{136 \bar{z}} \\
& A_{136 v}=A_{136 \bar{v}} \\
& A_{145 z}=A_{145 \bar{z}} \\
& A_{145 v}=-A_{145 \bar{v}} \\
& A_{235 z}=-A_{235 \bar{z}} \\
& A_{235 v}=A_{235 \bar{v}} \\
& A_{246 z}=-A_{246 \bar{z}} \\
& A_{246 v}=-A_{246 \bar{v}} .
\end{aligned}
$$

We can now use the additional condition:

$$
A_{135}+A_{146}+A_{236}+A_{245}=2 \Sigma A_{i}=2(A+B)=\mathrm{const}
$$

Thus $A$ has to be a constant what tells us, that there cannot be a "shape" deformation in $J$. Now we can use $\Sigma A_{i}=$ const again and write:

$$
\begin{aligned}
& A_{123456 z ; \bar{z} ; v ; \bar{v}}=\left(A_{136}+A_{245}\right)_{z ; \bar{z} ; v ; \bar{v}}=\left(A_{145}+A_{236}\right)_{z ; \bar{z} ; v ; \bar{v}} \\
& =\left(A_{235}+A_{146}\right)_{z ; \bar{z} ; v ; \bar{v}}=\left(A_{246}+A_{135}\right)_{z ; \bar{z} ; v ; \bar{v}}=0 .
\end{aligned}
$$

Using equation (14), it follows:

$$
A_{136}=A_{145}=A_{235}=A_{246}=\mathrm{const}
$$

That means that we also cannot have "shape" deformation in $\Omega$. The uplift of Iwasawa manifold to $G_{2}$ holonomy can only be done with "size" deformation. 


\subsection{Intrinsic torsion classes for M-theory with fluxes in 7-dimension with $S U(3)$ structure}

The difference compared to 6-dimensional case is the existence of a globally defined vector $v$. $S U(3)$ structure in $\mathrm{d}=7$ is then described by a triplet $v, J, \Omega$. The 2 -form $J$ now satisfies $J_{a}^{b} J_{b}^{c}=$ $-\delta_{a}^{c}+v_{a} v^{c}$. The metric of seven-dim space can be written as $d s_{7}{ }^{2}(x, t)=d s_{6}{ }^{2}(x, t)+v \otimes v$ with $v=e^{q \phi(x)} d t$. In 7 dimensions the decomposition of torsion gives us 15 classes:

$$
\tau \rightarrow R \oplus C_{1,2} \oplus V_{1,2} \oplus W_{1,2} \oplus S_{1,2} \oplus \mathcal{A}_{1,2} \oplus T
$$

where $C_{1,2}, W_{1,2}$ and $\mathrm{T}$ are complex.

After using necessary and sufficient conditions for obtaining $N=1$ supersymmetry solutions of $\mathrm{M}$ theory with fluxes, and comparing with the decomposition of the 4 -form $G$-flux given in terms of irreducible $S U(3)$ representations:

$$
G=-\frac{Q}{6} J \wedge J+J \wedge \mathcal{A}+\Omega_{-} \wedge V+v \wedge\left(\alpha_{1} \Omega_{+}+\alpha_{2} \Omega_{-}+J \wedge W+U\right),
$$

and the corresponding expression for $*_{7} G$ (that involves an additional flux component " $S$ ") we obtain:

$$
\begin{aligned}
R & =C_{1}=W_{1}=W_{2}=\mathcal{A}_{1}=T=S_{2}=0 ; \\
C_{2} & =\bar{C}_{2}=0 ; \\
V_{1} & =\frac{2}{3} V_{3}=\sigma ; \\
\mathcal{A}_{2} & =-\mathcal{A} \\
S_{1} & =-2 S .
\end{aligned}
$$

\subsection{Irreducible 7-manifold}

A naturally induced $S U(3)$-structure on seven-dimensional manifold without a killing isometry is given by:

$$
v=e^{q \phi} d t, J=\hat{J}(t), \Omega=\hat{\Omega}(t)
$$

where $t$ is variable parameterizing the interval $I$. For simplicity we first discuss $q=0$ :

$$
d s_{7}^{2}(y, t)=d s_{6}{ }^{2}(y, t)+d t^{2} .
$$

The conditions $\hat{d} J=-2 S \neq 0, \hat{d} \Omega=0$ give us that $M_{6}$ is a special-hermitian manifold,i.e. 
it is a complex non-Kähler manifold ${ }^{5}$. One can now build a 7 -manifold, that can be used in flux solutions of M-theory by solving differential equations in $t$. This construction generalizes the Hitchin's Flow equations for construction of $G_{2}$-holonomy manifold:

$$
\begin{aligned}
& \frac{\partial J}{\partial t}=\frac{2}{3} Q J-2 \mathcal{A} \\
& \frac{\partial \Omega}{\partial t}=Q \Omega .
\end{aligned}
$$

We now use an ansatz for $J$ with "shape" and "size" deformations:

$$
J=e^{a(t)} e^{12}+A(z, \bar{z}, v, \bar{v}) e^{34}+B(z, \bar{z}, v, \bar{v}) e^{56}
$$

where $\operatorname{Im}(A)=\operatorname{Im}(B)=0$.

$$
\begin{aligned}
\Rightarrow S= & -\frac{1}{2} \hat{d} J=-\frac{1}{2}\left[\left(e^{134}+i e^{234}\right) \frac{\partial A}{\partial z}+\left(e^{134}-i e^{234}\right) \frac{\partial A}{\partial \bar{z}}\right. \\
& +\left(e^{156}+i e^{256}\right) \frac{\partial B}{\partial z}+\left(e^{156}-i e^{256}\right) \frac{\partial B}{\partial \bar{z}}+\left(e^{356}+i e^{456}\right) \frac{\partial B}{\partial v} \\
& \left.+\left(e^{356}-i e^{456}\right) \frac{\partial B}{\partial \bar{v}}+B\left(-e^{135}+e^{245}+e^{146}+e^{236}\right)\right]
\end{aligned}
$$

The Bianchi identity reads:

$$
\begin{aligned}
& \frac{d Q}{6} \wedge J \wedge J+\frac{2}{9} Q^{2} J \wedge J \wedge v-\frac{4}{3} Q v \wedge J \wedge \mathcal{A}-\frac{Q}{3} J \wedge J \wedge \sigma \\
& +2 S \wedge \mathcal{A}+2 v \wedge \mathcal{A} \wedge \mathcal{A}+J \wedge \sigma \wedge \mathcal{A}-3 v \wedge \sigma \wedge J \wedge W \\
& -2 v \wedge S \wedge W+v \wedge J \wedge d W+d * S \stackrel{?}{=} 0
\end{aligned}
$$

where the question mark over the equality sign is indicative of the possibility that one can not satisfy a source-free Bianchi identity (as will be the case).

With $Q J=\frac{3}{2}[\dot{J}+2 A] \Rightarrow Q=\frac{3}{2} \dot{a}(t)$ it follows:

$$
\begin{aligned}
& \frac{d Q}{6} \wedge J \wedge J=\frac{\ddot{a}}{2} d t \wedge\left[e^{a} A e^{1234}+e^{a} B e^{1256}+A B e^{3456}\right] \\
& \frac{2}{9} Q^{2} J \wedge J \wedge v=\dot{a}^{2} d t \wedge\left[e^{a} A e^{1234}+e^{a} B e^{1256}+A B e^{3456}\right]
\end{aligned}
$$

${ }^{5}$ As the torsion classes $W_{1}$ and $W_{2}$ are defined via: $\left(\hat{d} \hat{\Omega}_{ \pm}\right)^{(2,2)}=W_{1}^{ \pm \wedge} \wedge \hat{J}+W_{2}^{ \pm} \hat{J}$, this implies $W_{1}=W_{2}=0$, implying the vanishing of the Nijenhuis tensor. Hence the manifold is a complex manifold. Further, $W_{5}=\frac{1}{2} \hat{\Omega_{+}} \mid \hat{d} \hat{\Omega_{+}}=0$. As $W_{1}$ is also identified with $[\hat{d} \hat{J}]^{(3,0)}$ and given that $W_{1}=0$ this implies that $d J \in \Lambda^{(2,1)} \oplus \Lambda^{(1,2)}\left[\right.$ As $\hat{d} \hat{J} \neq 0$, writing $\alpha^{(p, q)}$ in terms of a real $\alpha^{p+q}: \alpha_{m_{1} \ldots . . m_{p+q}}^{(p, q)}=$ $\left.\left.\left(P^{+}\right)_{m_{1} \ldots\left(P^{+}\right.}^{n_{1}}\right)_{m_{p}}^{n_{p}}\left(P^{-}\right)_{m_{p+1} \ldots .}^{n_{p+1} \ldots\left(P^{-}\right.}\right)_{m_{p+q}}^{n_{p+q}} \alpha_{n_{1} \ldots n_{p+q}}^{p+q}$, where $\left(P^{ \pm}\right)_{m}^{n} \equiv \frac{1}{2}\left(\delta_{m}^{n} \pm i J_{m}^{n}\right)$, this implies that $\hat{d} \alpha^{(p, q)} \in$ $\Lambda^{(p+2, q-1)} \oplus \Lambda^{(p+1, q)} \oplus \Lambda^{(p, q+1)} \oplus \Lambda^{(p-1, q+2)}[15]$, where $\left.\alpha^{(p, q)} \in \Lambda^{(p, q)}.\right]$. Further, as $W_{3}$ is identified with $[\hat{d} \hat{J}]^{(2,1)}$ and $\hat{d} \hat{J}^{(2,1)}=\left(\hat{J} \wedge W_{4}\right)^{(2,1)}+W_{3}$, one concludes that $W_{4}=0$. Thus, the six-fold is a special hermitian manifold. 
And with $\mathcal{A} \equiv \frac{1}{2} \dot{a}(t)\left[A(z, \bar{z}, v, \bar{v}) e^{34}+B(z, \bar{z}, v, \bar{v}) e^{56}\right]$ it follows:

$$
\begin{aligned}
& -\frac{4}{3} Q v \wedge J \wedge A=-\dot{a}^{2} d t \wedge\left[e^{a} A e^{1234}+e^{a} B e^{1256}+2 A B e^{3456}\right] \\
& 2 S \wedge \mathcal{A}=-\frac{\dot{a}(t)}{2}\left[e^{13456}\left(\frac{\partial}{\partial z}+\frac{\partial}{\partial \bar{z}}\right)(A B)+i e^{23456}\left(\frac{\partial}{\partial z}-\frac{\partial}{\partial \bar{z}}\right)(A B)\right] \\
& 2 v \wedge \mathcal{A} \wedge \mathcal{A}=\dot{a}^{2} A B d t \wedge e^{3456} \\
& d \star_{7} S=-\frac{1}{2} d t \wedge\left[2 e^{1256}\left(\frac{\partial^{2} A}{\partial z^{2}}+\frac{\partial^{2} A}{\partial \bar{z}^{2}}\right)\right. \\
& +2 e^{1234}\left(\frac{\partial^{2} B}{\partial z^{2}}+\frac{\partial^{2} B}{\partial \bar{z}^{2}}+\frac{\partial^{2} B}{\partial v^{2}}+\frac{\partial^{2} B}{\partial \bar{v}^{2}}+2 B\right) \\
& -e^{2356}\left(\frac{\partial^{2} A}{\partial z \partial v}+\frac{\partial^{2} A}{\partial \bar{z} \partial v}+c . c .\right)-i e^{2456}\left(\frac{\partial^{2} A}{\partial z \partial v}+\frac{\partial^{2} A}{\partial \bar{z} \partial v}-c . c .\right) \\
& -i e^{1356}\left(\frac{\partial^{2} A}{\partial z \partial v}-\frac{\partial^{2} A}{\partial \bar{z} \partial v}-c . c .\right)+e^{1456}\left(\frac{\partial^{2} A}{\partial z \partial v}-\frac{\partial^{2} A}{\partial \bar{z} \partial v}+c . c .\right) \\
& i\left(e^{1346}+e^{2345}\right)\left(\frac{\partial B}{\partial v}-\frac{\partial B}{\partial \bar{v}}\right)-\left(e^{1345}-e^{2346}\right)\left(\frac{\partial B}{\partial v}+\frac{\partial B}{\partial \bar{v}}\right) \\
& \quad-i\left(e^{1245}+e^{1236}\right)\left[\left(\frac{\partial B}{\partial z}-\frac{\partial B}{\partial \bar{z}}\right)+\left(\frac{\partial A}{\partial z}-\frac{\partial A}{\partial \bar{z}}\right)\right] \\
& \left.+i\left(-e^{1246}+e^{1235}\right)\left[\left(\frac{\partial B}{\partial z}+\frac{\partial B}{\partial \bar{z}}\right)-\left(\frac{\partial A}{\partial z}+\frac{\partial A}{\partial \bar{z}}\right)\right]\right]
\end{aligned}
$$

The Hodge dual of $d G$ then reads:

$$
\begin{aligned}
& \star_{11} d G=e^{4 \Delta} d x^{0} \wedge d x^{1} \wedge d x^{2} \wedge d x^{3} \wedge \\
& {\left[\left(\frac{\ddot{a}}{2} e^{a} A-\left(\frac{\partial^{2} B}{\partial z^{2}}+\frac{\partial^{2} B}{\partial \bar{z}^{2}}+\frac{\partial^{2} B}{\partial v^{2}}+\frac{\partial^{2} B}{\partial \bar{v}^{2}}+2 B\right)\right) e^{56}\right.} \\
+ & \left(\frac{\ddot{a}}{2} e^{a} B-\left(\frac{\partial^{2} A}{\partial z^{2}}+\frac{\partial^{2} A}{\partial \bar{z}^{2}}\right)\right) e^{34}+\frac{\ddot{a}}{2} A B e^{12}-\frac{\dot{a}(t)}{2}\left(\frac{\partial}{\partial z}+\frac{\partial}{\partial \bar{z}}\right) A B d t \wedge e^{2} \\
& -i \frac{\dot{a}(t)}{2}\left(\frac{\partial}{\partial z}-\frac{\partial}{\partial \bar{z}}\right) A B d t \wedge e^{1} \\
& -\frac{1}{2}\left[-e^{14}\left(\frac{\partial^{2} A}{\partial z \partial v}+\frac{\partial^{2} A}{\partial \bar{z} \partial v}+c . c .\right)-i e^{13}\left(\frac{\partial^{2} A}{\partial z \partial v}+\frac{\partial^{2} A}{\partial \bar{z} \partial v}-\text { c.c. }\right)\right. \\
& -i e^{24}\left(\frac{\partial^{2} A}{\partial z \partial v}-\frac{\partial^{2} A}{\partial \bar{z} \partial v}-c . c .\right)+e^{23}\left(\frac{\partial^{2} A}{\partial z \partial v}-\frac{\partial^{2} A}{\partial \bar{z} \partial v}+c . c .\right) \\
& i\left(e^{25}+e^{16}\right)\left(\frac{\partial B}{\partial v}-\frac{\partial B}{\partial \bar{v}}\right)-\left(e^{26}-e^{15}\right)\left(\frac{\partial B}{\partial v}+\frac{\partial B}{\partial \bar{v}}\right)
\end{aligned}
$$




$$
\begin{aligned}
& -i\left(e^{36}+e^{45}\right)\left[\left(\frac{\partial B}{\partial z}-\frac{\partial B}{\partial \bar{z}}\right)+\left(\frac{\partial A}{\partial z}-\frac{\partial A}{\partial \bar{z}}\right)\right] \\
& \left.\left.+i\left(-e^{35}+e^{46}\right)\left[\left(\frac{\partial B}{\partial z}+\frac{\partial B}{\partial \bar{z}}\right)-\left(\frac{\partial A}{\partial z}+\frac{\partial A}{\partial \bar{z}}\right)\right]\right]\right]
\end{aligned}
$$

We use this form because $d G=\star_{11} \mathcal{I}_{6}$, the six-form $\mathcal{I}_{6}$ specifying the position of the $M 5$-branes transverse to the world volume, when one interprets the metric to represent $M 5$-branes wrapped around two-cycles (with densities $\rho_{i j}$ ) (See [1]). Computing $\star_{11} d G$ we obtain informations about $\mathcal{I}_{6}$.

We now simplify in the following way: $A, B$ do not depend on $v$ and $\bar{v} ; A=B ; \frac{\partial A}{\partial z}=\frac{\partial A}{\partial \bar{z}}$. One parameter family of solution satisfying the assumptions and the requirement of periodicity in the $T^{2}$-valued $z$, is given by $A(z, \bar{z})=\cos [m \pi(z+\bar{z})]$ where $m \in \mathbf{Z}$.

The Hodge dual of $d G$ then reads:

$$
\begin{aligned}
& \star_{11} d G=e^{4 \Delta} d x^{0} \wedge d x^{1} \wedge d x^{2} \wedge d x^{3} \wedge \\
& {\left[\left(\frac{\ddot{a}}{2} e^{a}+2(m \pi)^{2}-2\right) A e^{56}+\left(\frac{\ddot{a}}{2} e^{a}+2(m \pi)^{2}\right) A e^{34}+\frac{\ddot{a}}{2} A^{2} e^{12}-2 \alpha \dot{a} \frac{\partial A}{\partial z} e^{2 t}\right]} \\
& \equiv e^{4 \Delta} d x^{0} \wedge d x^{1} \wedge d x^{2} \wedge d x^{3} \wedge\left(\rho_{12} e^{12}+\rho_{34} e^{34}+\rho_{56} e^{56}+\rho_{2 t} e^{2 t}\right)
\end{aligned}
$$

Interestingly, along hypersurfaces given by: $z+\bar{z}=\frac{(2 k+1)}{2 m}$, one gets a source-free Bianchi identity. For other points, we consider now different cases for $\star_{11} d G$ :

- $a=$ const, which would mean that we do not have "size" deformation. $\Rightarrow \star_{11} d G=$ $\left.-2 \alpha^{2} A e^{34}+2(m \pi)^{2}-2\right) A e^{56}$ That means the densities $\rho_{34}$ and $\rho_{56}$ are not zero; it is the only posibility that two terms vanish.

- $\dot{a}(t)=$ const: $\rho_{12}=0$

- We can also require that $\rho_{34}=0$ - we then have non-zero density $\rho_{12}$ with $a(t)$ given by: $a(t)=\ln \left[\frac{e^{-\gamma_{1} t+\gamma_{2}}\left(-32(m \pi)^{2}+e^{\gamma_{1} t+\gamma_{2}}\right)^{2}}{16 \gamma_{1}^{2}}\right], \gamma_{1,2}$ being constants of integration. One can get a similar expression for $a(t)$ if one sets $\rho_{56}=0$.

The Warp-factor can now be calculated to:

$$
\triangle(t)=-\frac{1}{2} a(t)+\text { const; set const }=0
$$

For the metric it follows:

$$
d s_{11}^{2}=e^{-a(t)} \eta_{\mu \nu} d x^{\mu} d x^{\nu}+d s_{7}{ }^{2} .
$$




\subsection{Discussion for $q \neq 0$}

For the 7-dimensional metric we assume $d s_{7}{ }^{2}(y, t)=e^{p \phi} d s_{6}{ }^{2}(y, t)+e^{2 \phi} d t^{2}$. One can also show the following relations:

$$
\sigma \equiv \hat{d} \triangle=-\frac{1}{2} \hat{d} \phi ; \quad \dot{\triangle}=-\frac{1}{3} Q e^{\phi}
$$

Unlike what is shown in [1], the following equation is identically fullfilled:

$$
3 d \sigma=d Q \wedge v+2 Q v \wedge \sigma
$$

The two- and the three-form of SU(3) structure on the six-fold are now:

$$
J=e^{p \phi} \hat{J} ; \quad \Omega=e^{\frac{3}{2} p \phi} \hat{\Omega} .
$$

Thus, one gets a non-Kähler complex manifold referred to as a balanced manifold ${ }^{6}$. We obtain:

$$
\hat{d} \hat{J}=-2 e^{-\frac{1}{2} \phi} S ; \quad \hat{d} \hat{\Omega}=\frac{3}{4} \hat{d} \phi \wedge \hat{\Omega}
$$

and for the Flow equations it follows:

$$
\begin{aligned}
& \frac{\partial \hat{J}}{\partial t}=\frac{2}{3} e^{\phi} Q \hat{J}-2 e^{\frac{\phi}{2}} \mathcal{A}-\frac{1}{2} \dot{\phi} \hat{J} \\
& \frac{\partial \hat{\Omega}}{\partial t}=Q e^{\phi} \hat{\Omega}-\frac{3}{4} \dot{\phi} \hat{\Omega} .
\end{aligned}
$$

For simplicity we choose $p=\frac{1}{2}$. We assume $A=\frac{1}{2} e^{-\phi} \dot{a}\left(A e^{34}+B e^{56}\right)$ from where it follows $Q=\frac{3}{2} \dot{a} e^{-\phi}$. Hence, $\Delta=-\frac{1}{2} a(t)-\frac{1}{2} \phi$. Through comparison of the coefficients using $\sigma=-\frac{1}{2} \hat{d} \phi=$ $\frac{2}{3} W_{-} \mid J$ we obtain :

$$
W=-\frac{3}{4}\left[e^{1} i\left(\phi_{z}-\phi_{\bar{z}}\right) e^{-a}-e^{2}\left(\phi_{z}+\phi_{\bar{z}}\right) e^{-a}+e^{3} i \frac{\left(\phi_{v}-\phi_{\bar{v}}\right)}{A}-e^{4} \frac{\left(\phi_{v}+\phi_{\bar{v}}\right)}{A}\right] .
$$

Now we can calculate the terms for the Bianchi identity. The same is done in Appendix A. The $D=11$-Hodge dual of $d G$ can then be calculated, as done towards the end of Appendix A. Now, using the simplifications we already used for $\mathrm{q}=0$ : $A, B$ do not depend on $v$ and $\bar{v}$; $A=B ; \frac{\partial A}{\partial z}=\frac{\partial A}{\partial \bar{z}}$, thereby obtaining a one-parameter family of solution satisfying the assumptions

\footnotetext{
${ }^{6}$ This time, $W_{5}=\frac{1}{2} \hat{\Omega_{+-}} \mid \hat{d} \hat{\Omega_{+}}=\frac{3}{8} \hat{d} \phi \neq 0$. Hence, the torsion $\tau \in W_{3} \oplus W_{5}$ - a balanced manifold.
} 
$A(z, \bar{z})=\cos [m \pi(z+\bar{z})], m \in \mathbf{Z}$, and additionally assuming $\phi$ does not depend on $v$ and $\bar{v}$, the eleven-dimensional Hodge dual of $d G$ then reads:

$$
\begin{aligned}
& \star_{11} d G=e^{4 \Delta} d x^{0} \wedge d x^{1} \wedge d x^{2} \wedge d x^{3} \wedge \\
& {\left[\left(e^{-\phi} \frac{\ddot{a}}{2} e^{a}-2 \alpha^{2}-2+\frac{\phi_{z z}+\phi_{\bar{z} \bar{z}}}{2}+\alpha\left(\phi_{z}+\phi_{\bar{z}}\right)+\frac{3}{2} e^{\phi} e^{-a} \alpha\left(\phi_{z}+\phi_{\bar{z}}\right)-\frac{1}{2} \dot{a} \dot{\phi} e^{-\phi}\right) A e^{56}\right.} \\
+ & \left(e^{-\phi} \frac{\ddot{a}}{2} e^{a}-2 \alpha^{2}+\frac{\phi_{z z}+\phi_{\bar{z} \bar{z}}}{2}+\alpha\left(\phi_{z}+\phi_{\bar{z}}\right)+\frac{3}{2} e^{\phi} e^{-a} \alpha\left(\phi_{z}+\phi_{\bar{z}}\right)-\frac{1}{2} \dot{a} \dot{\phi} e^{-\phi}\right) A e^{34} \\
+ & \left(\frac{\ddot{a}}{2}-\frac{1}{2} \dot{a} \dot{\phi} e^{-\phi}\right) A^{2} e^{12}+\frac{A}{4}\left(\phi_{z}+\phi_{\bar{z}}\right)\left[3 e^{\phi} e^{-a}-1\right]\left(e^{35}-e^{46}\right) \\
+ & \left.\frac{A}{4}\left(\phi_{z}-\phi_{\bar{z}}\right)\left[3 e^{\phi} e^{-a}-1\right] i\left(e^{36}+e^{45}\right)-2 \alpha \dot{a} e^{-\phi} A^{2} d t \wedge e^{2}\right] \\
& \equiv \operatorname{vol} \text { form }\left(\mathbf{R}^{3,1}\right)\left(\rho_{12} e^{12}+\rho_{34} e^{34}+\rho_{56} e^{56}+\rho_{35} e^{35}+\rho_{36} e^{36}+\rho_{45} e^{45}+\rho_{46} e^{46}+\rho_{2 t} e^{2 t}\right) .
\end{aligned}
$$

Generically, one thus sees, that the deformed Iwasawa is a balanced manifold. However, if one assumes that $\hat{d} \phi=0$ and $\phi=\phi(t)$, then the balanced six-fold becomes a special-hermitian six-fold. In such a situation, $\rho_{35}=\rho_{36}=\rho_{45}=\rho_{46}=0$. Again, along hypersurfaces given by: $z+\bar{z}=\frac{(2 k+1)}{2 m}$, one gets a source-free Bianchi identity.

For a balanced manifold, assuming $\dot{\phi}=0, \phi_{z}=-\phi_{\bar{z}}$, which is satisfied by, e.g., $\phi=$ $\ln [\cos [i n \pi(z-\bar{z})]], n \in \mathbf{Z}$ (once again ensuring periodicity w.r.t. the $T^{2}$-valued $z$ ), one gets the following metrics for seven-folds with $S U(3)$ structure:

- having frozen the "size" deformation of the Iwasawa, the seven-fold is a fibration of an interval over a balanced six-fold:

$$
d s_{7}^{2}=(\cos [2 n \pi \operatorname{Im}(z)])^{2} d t^{2}+|d z|^{2}+\cos [2 m \pi \operatorname{Re}(z)]|d v|^{2}+\cos [2 m \pi \operatorname{Re} z]|d u-v d z|^{2},
$$

which gives

$$
d s_{11}^{2}=\sec [2 n \pi \operatorname{Im}(z)] d s_{\mathbf{R}^{3,1}}^{2}+d s_{7}^{2} ;
$$

this implies that $\rho_{12}=\rho_{t 2}=0$. The interval, for this case, could also be replaced by $S^{1}$ as periodicity w.r.t. this $S^{1}$ will then not be a problem.

- For the six-folds to be special-hermitian manifolds by assuming $\phi=\phi(t)=a(t)$ (i.e., $\hat{d} \phi=0)$, and further $\ddot{a}-(\dot{a})^{2}=2-2(m \pi)^{2}$, solved by $a(t)= \pm\left(\sqrt{2(m \pi)^{2}-2}\right) t+$ constant, implying $\rho_{56}=0$, one gets the seven-fold to be a warped product of a balanced manifold and an interval:

$$
\begin{aligned}
d s_{7}^{2}= & e^{-2\left(\sqrt{2(m \pi)^{2}-2}\right) t+\gamma_{3}} d t^{2}+e^{-\left(\sqrt{2(m \pi)^{2}-2}\right) t+\frac{\gamma_{3}}{2}}|d z|^{2}+\cos [2 m \pi \operatorname{Re}(z)]|d v|^{2} \\
& +\cos [2 m \pi \operatorname{Re}(z)]|d u-v d z|^{2},
\end{aligned}
$$


which gives a two $\left.\left(m \in \mathbf{Z}, \gamma_{3} \in \mathrm{R}\right)\right)$-parameter family of solutions:

$$
d s_{11}^{2}=e^{\left(\sqrt{2(m \pi)^{2}-2}\right) t-\frac{\gamma_{3}}{2}} d s_{\mathbf{R}^{3,1}}^{2}+d s_{7}^{2} .
$$

\section{Interpretation of the Uplifts}

For uplifts to seven-folds with $S U(3)$ structure, as $d G$, generically, is not zero, we only have solutions with sources. The non-zero piece of $d G$, for $q=0$, in the ansatz we worked with, could be interpreted due to $M 5$-branes wrapping 2-cycles with respect to the standard complex structure in the internal seven-fold viewed as a warped product of a (special hermitian or balanced) six-fold and the internal. For $q \neq 0$, in general, one ends up with balanced manifolds. However, the issue of supersymmetry of the wrapped M5-branes should be looked into more carefully, by , e.g., looking at the world-volume theory of these branes ${ }^{7}$ to ensure that the wrapped $M 5$-branes that minimize the energy functional, belong to $M$-theory.

The two-cycle is calibrated w.r.t. the generalized calibration $[16,17] J$ thereby guaranteeing the minimization of the corresponding energy functional, but away from the standard-complexstructure point in the moduli space of almost complex structures on the Iwasawa. At any generic point in the moduli space of almost complex structures on the Iwasawa manifold, the two form $J$ can be described by using a basis constructed of $S O(4)$ matrices $P^{i}$ and the one forms $e_{i}$ of the orthonormal basis [13]:

$$
J=\frac{i}{2}(\alpha \wedge \bar{\alpha}+\beta \wedge \bar{\beta}+\gamma \wedge \bar{\gamma})
$$

where:

$$
\begin{aligned}
& \alpha \equiv \cos \theta f^{1}-\sin \theta e^{6}+i f^{2} \\
& \beta \equiv f^{3}+i f^{4} \\
& \gamma \equiv+i\left(\cos \theta e^{6}-\sin \theta f^{1}+i f^{2}\right)
\end{aligned}
$$

and $f^{i}=P^{i}{ }_{j} e^{j}, P \in S O(4)$.

The wedge product of two different $f \mathrm{~s}$ is defined as:

$$
f^{i} \wedge f^{j}=\frac{1}{2} P_{[k}^{i} P^{j}{ }_{l]} e^{k} \wedge e^{l} .
$$

This means that we cannot have a non-trivial "shape" (and "size") deformation using the standard complex structure. By turning on fluxes one moves away from the standard complex structure. The moduli space is then neither "edge" nore standard complex structure with respect to the one forms $e_{i}$ of the orthonormal basis.

\footnotetext{
${ }^{7} \mathrm{AM}$ thanks O.Lunin for a discussion on this point.
} 
If one had used a singular ansatz for $J$ implying localization w.r.t. directions transverse to the $M 5$-brane world volume, and such that $e^{2 \Delta}$ vanished at the location of the $M 5$-branes ${ }^{8}$, then using the results of [18], one could interpret the 11-dimensional metric after uplifting the balanced manifold to a seven-fold with $S U(3)$ structure, as corresponding to $M 5$ brane wrapped around a Riemann surface in $\mathbf{C}^{3}$ :

$$
d s_{11}^{2}=H_{1}^{2} d s_{\mathbf{R}^{3,1}}^{2}+g_{m \bar{n}} d z^{m} d \bar{z}^{\bar{n}}+H_{2}^{2} d t^{2},
$$

one gets the required interpretation by setting $H_{1}=\Delta$ and $H_{2}=\phi$ (See [1]) and regarding $z, v, u$ as the complex coordinates on $\mathbf{C}^{3}$.

Now, as an interesting mathematical curiosity, using the results of [2], we will show that one can associate a Riemann surface to a choice of the "shape" deformation functions. Consider the system of partial differential equations:

$$
\frac{\partial A}{\partial z}=\phi B ; \frac{\partial B}{\partial \bar{z}}=-\phi A,
$$

which is equivalent to:

$$
\frac{\partial^{2} B}{\partial z \partial \bar{z}}=\frac{1}{\phi} \frac{\partial \phi}{\partial z} \frac{\partial B}{\partial \bar{z}}-\phi^{2} B
$$

(or one could switch $B$ with $A$ and $z \leftrightarrow \bar{z}$ ). We see that the following choice solves (44):

$$
\begin{array}{ll}
A_{1}(z, \bar{z})=\sin [m \pi(z+\bar{z})], & B_{1}(z, \bar{z})=\cos [m \pi(z+\bar{z})], \quad \phi(z, \bar{z})=m \pi \\
A_{1}(z, \bar{z})=\cos [m \pi(z+\bar{z})], & B_{1}(z, \bar{z})=\sin [m \pi(z+\bar{z})], \phi(z, \bar{z})=-m \pi .
\end{array}
$$

One can construct an $\mathbf{R}^{3}$ with coordinates::

$$
\begin{aligned}
& X^{1}+i X^{2}=i \int_{\Gamma}\left(A_{1}^{2} d z^{\prime}-B_{1}^{2} d \bar{z}^{\prime}\right), \\
& X^{1}-i X^{2}=i \int_{\Gamma}\left(B_{1}^{2} d z^{\prime}-A_{1}^{2} d \bar{z}^{\prime}\right), \\
& X^{3}=-\int_{\Gamma}\left(A_{1} B_{1} d z^{\prime}+A_{1} B_{1} d \bar{z}^{\prime}\right),
\end{aligned}
$$

and for the contour $\Gamma=(x, y) \mid x=y, 0<x<1$,

$$
\begin{aligned}
& X^{1}+i X^{2}=-x-\frac{i}{4 m \pi} \sin (4 m \pi x), \\
& X^{3}=\frac{\cos (4 m \pi x)-1}{2 m \pi},
\end{aligned}
$$

\footnotetext{
${ }^{8} \mathrm{AM}$ thanks J.Maldacena for emphasizing this point to him.
} 
which would be the Weierstraß representation for the conformal immersion in $\mathbf{R}^{3}$ with the condition of conformal immersion (implying $g_{z z}=g_{\bar{z} \bar{z}}=0$ ):

$$
\left(\frac{\partial X^{1}}{\partial z}\right)^{2}+\left(\frac{\partial X^{2}}{\partial z}\right)^{2}+\left(\frac{\partial X^{3}}{\partial z}\right)^{2}=0
$$

a quadric in $\mathbf{C P}^{2}$. The induced metric on the Riemann surface is given by:

$$
d s^{2}=\left(A_{1}^{2}+B_{1}^{2}\right)^{2}|d z|^{2}=|d z|^{2},
$$

which is just a $T^{2}$. In appendix $\mathrm{B}$, we discuss a Weierstraß representation of conformal immersion in $\mathbf{C}^{3}$ for the linear dilaton ansatz but for solutions that are valid only locally because of lack of periodicity w.r.t. the $T^{2}$-valued coordinates.

\section{Conclusion}

We uplifted the Iwasawa to a $G_{2}$-manifold via Hitchin's Flow equations using "size"-deformations, and found that an uplift via Hitchin's flow equations (to a seven-fold with $G_{2}$ holonomy) with non-trivial "shape" deformation is not possible. Furthermore we uplifted the Iwasawa to an irreducible 7 manifold with $S U(3)$-structure through deforming the metric using "shape" and "size"-deformations via generalization of the Hitchin's Flow equations. Without simplification the seven-fold of $S U(3)$ structure turns out to be a warped product of either a special hermtian or a balanced six-fold and an interval (which for cases when one freezes the "size deformations", could also be replaced by an $S^{1}$ ). The uplifted metric could be interpreted as $M 5$-branes wrapping two-cycles calibrated by the generalized calibration (like [1]), the two-form corresponding to the almost complex structure, but away from the standard complex structure point (in the moduli space of almost complex structures on the Iwasawa). The supersymmetry of these wrapped membranes needs to be further looked into. We also showed how to associate a Riemann surface (via Weierstraß representation for conformal immersion in $\mathbf{R}^{l}$ ) with the "shape deformation" functions of seven-folds with $S U(3)$ structure and the dilaton. The relationship of the same to, e.g., supersymmetric two-cycles or Riemann surfaces embedded in seven-folds of $S U(3)$ structure around which $M 5$-branes wrap, needs to be understood.

However, if one allows for sources, as was the case, then there seems to be the possibility of considering singular uplifts of the following type. Given that it is possible to im-

pose just the requirement of (anti-)analyticity on the (anti-)holomorphic parts of the functions $A(z, \bar{z} ; v, \bar{v}), B(z, \bar{z} ; v, \bar{v})$ and $\phi(z, \bar{z} ; v, \bar{v})$, remembering that the complex coordinates $z, v$ are $T^{2}$ valued, one can hence introduce the doubly periodic functions: the Weierstraß elliptic function with double poles and the Jacobi elliptic function with simple poles, into the metric of the sevenfold. The elliptic surface corresponding to the Weierstraß elliptic function could perhaps be related to the Riemann surface relevant to the world volume of the $M 5$-brane relevant to the uplifts. See [19] for some connections relevant to this study. 


\section{Acknowledgements}

AF would like to thank the physics department of IIT Roorkee for the hospitality during her stay there as part of her semester-long study leave from RWTH, Aachen, and the organizers of THEPI (2005) at IIT Roorkee where some preliminary results of this work were presented. One of us (AM) would like to thank S.Chiossi, G.Papadopoulos and J.Gauntlett for useful communications, and especially J.Maldacena for critical comments on the material of the first version of this paper and O.Lunin for discussions, the Harvard high energy theory group (and S.Minwalla in particular) and IAS, Princeton for the hospitality during his stay there where part of this work was completed, the Department of Atomic Energy (Board of Research in Nuclear Sciences), Govt. of India for a research grant (under the DAE young scientist award scheme) and the organizers of PASCOS05, Gyeongju, Korea, where some preliminary results of this work were presented. We thank H.S.Solanki for participation in the initial stages of this project.

\section{A The Bianchi Identity for $q \neq 0$}

In this appendix, we discuss the evaluation of the various terms in the Bianchi identity.

$$
\begin{aligned}
& \frac{d Q}{6} \wedge J \wedge J=\frac{e^{-\phi}}{2}\left[\ddot{a}\left(e^{a} A e^{1234 t}+e^{a} B e^{1256 t}+A B e^{3456 t}\right)\right. \\
& -\dot{a}\left[\left(\phi_{z}+\phi_{\bar{z}}\right) A B e^{13456}+i\left(\phi_{z}-\phi_{\bar{z}}\right) A B e^{23456}+\left(\phi_{v}+\phi_{\bar{v}}\right) e^{a} B e^{12356}\right. \\
& \left.+i\left(\phi_{v}-\phi_{\bar{v}}\right) e^{a} B e^{12456}\right]+\dot{\phi}\left[e^{a} A e^{1234 t}+e^{a} B e 1256 t+A B e^{3456 t}\right] \\
& \frac{2}{9} Q^{2} J \wedge J \wedge v=\dot{a}^{2} e^{-\phi} d t \wedge\left[e^{a} A e^{1234}+e^{a} B e^{1256}+A B e^{3456}\right] \\
& -\frac{4}{3} Q v \wedge J \wedge A=-\dot{a}^{2} e^{-\phi} d t \wedge\left[e^{a} A e^{1234}+e^{a} B e^{1256}+2 A B e^{3456}\right] \\
& -\frac{Q}{3} J \wedge J \wedge \sigma=\frac{\dot{a}}{2} e^{-\phi}\left[\left(\phi_{z}+\phi_{\bar{z}}\right) A B e^{13456}+i\left(\phi_{z}-\phi_{\bar{z}}\right) A B e^{23456}\right. \\
& \left.\left.+\left(\phi_{v}+\phi_{\bar{v}}\right) e^{a} B e^{12356}+i\left(\phi_{v}-\phi_{\bar{v}}\right) e^{a} B e^{12456}\right]\right] \\
& 2 S \wedge \mathcal{A}=-\frac{\dot{a}}{2} e^{-\phi}\left[e^{13456}\left[\left(\frac{\partial}{\partial z}+\frac{\partial}{\partial \bar{z}}\right) A B-\left(\phi_{z}+\phi_{\bar{z}}\right) A B\right]\right. \\
& +i e^{23456}\left[\left(\frac{\partial}{\partial z}-\frac{\partial}{\partial \bar{z}}\right) A B-\left(\phi_{z}-\phi_{\bar{z}}\right) A B\right] \\
& \left.-e^{12356} \frac{e^{a}}{2} B\left(\phi_{v}+\phi_{\bar{v}}\right)-e^{12456} i \frac{e^{a}}{2} B\left(\phi_{v}-\phi_{\bar{v}}\right)\right]
\end{aligned}
$$




$$
2 v \wedge \mathcal{A} \wedge \mathcal{A}=e^{-\phi} \dot{a}^{2} A B d t \wedge e^{3456}
$$

$$
\begin{aligned}
& J \wedge \sigma \wedge \mathcal{A}=-\frac{\dot{a}}{2} e^{-\phi}\left[\left(\phi_{z}+\phi_{\bar{z}}\right) A B e^{13456}+i\left(\phi_{z}-\phi_{\bar{z}}\right) A B e^{23456}+\left(\phi_{v}+\phi_{\bar{v}}\right) \frac{e^{a}}{2} B e^{12356}\right. \\
& \left.+i\left(\phi_{v}-\phi_{\bar{v}}\right) \frac{e^{a}}{2} B e^{12456}\right] \\
& -3 v \wedge \sigma \wedge J \wedge W=-\frac{9}{8} e^{\phi}\left[-e^{1234 t}\left[A e^{-a}\left(4 \phi_{z} \phi_{\bar{z}}\right)+\frac{e^{a}}{A}\left(4 \phi_{v} \phi_{\bar{v}}\right)\right]-e^{1256 t} B e^{-a} 4 \phi_{z} \phi_{\bar{z}}\right. \\
& +e^{1356 t} i B\left[-\left(\phi_{z}-\phi_{\bar{z}}\right)\left(\phi_{v}+\phi_{\bar{v}}\right) e^{-a}+\frac{1}{A}\left(\phi_{z}+\phi_{\bar{z}}\right)\left(\phi_{v}-\phi_{\bar{v}}\right)\right] \\
& +e^{1456 t} B\left[\left(\phi_{z}-\phi_{\bar{z}}\right)\left(\phi_{v}-\phi_{\bar{v}}\right) e^{-a}-\frac{1}{A}\left(\phi_{z}+\phi_{\bar{z}}\right)\left(\phi_{v}+\phi_{\bar{v}}\right)\right] \\
& +e^{2356 t} B\left[\left(\phi_{z}+\phi_{\bar{z}}\right)\left(\phi_{v}+\phi_{\bar{v}}\right) e^{-a}-\frac{1}{A}\left(\phi_{z}-\phi_{\bar{z}}\right)\left(\phi_{v}-\phi_{\bar{v}}\right)\right] \\
& +e^{2456 t} i B\left[\left(\phi_{z}+\phi_{\bar{z}}\right)\left(\phi_{v}-\phi_{\bar{v}}\right) e^{-a}-\frac{1}{A}\left(\phi_{z}-\phi_{\bar{z}}\right)\left(\phi_{v}+\phi_{\bar{v}}\right)\right] \\
& \left.-e^{3456 t} \frac{B}{A} 4 \phi_{v} \phi_{\bar{v}}\right]
\end{aligned}
$$

$$
\begin{aligned}
& -2 v \wedge S \wedge W=-\frac{3}{4} e^{\phi} d t \wedge\left[e^{1234}\left[2 e^{-a}\left[-A_{z} \phi_{\bar{z}}-A_{\bar{z}} \phi_{z}+A \phi_{z} \phi_{\bar{z}}\right]+\frac{e^{a}}{A} 2 \phi_{v} \phi_{\bar{v}}\right]\right. \\
& +e^{1256} 2 e^{-a}\left[-B_{z} \phi_{\bar{z}}-B_{\bar{z}} \phi_{z}+B \phi_{z} \phi_{\bar{z}}\right]+e^{3456} \frac{2}{A}\left[-B_{v} \phi_{\bar{v}}-B_{\bar{v}} \phi_{v}+B \phi_{v} \phi_{\bar{v}}\right]
\end{aligned}
$$$$
+i e^{1356}\left[\frac{\left(\phi_{v}-\phi_{\bar{v}}\right)}{A}\left[\left(B_{z}+B_{\bar{z}}\right)-\frac{B}{2}\left(\phi_{z}+\phi_{\bar{z}}\right)\right]-e^{-a}\left(\phi_{z}-\phi_{\bar{z}}\right)\left[\left(B_{v}+B_{\bar{v}}\right)-\frac{B}{2}\left(\phi_{v}+\phi_{\bar{v}}\right)\right]\right]
$$$$
-e^{1456}\left[\frac{\left(\phi_{v}+\phi_{\bar{v}}\right)}{A}\left[\left(B_{z}+B_{\bar{z}}\right)-\frac{B}{2}\left(\phi_{z}+\phi_{\bar{z}}\right)\right]-e^{-a}\left(\phi_{z}-\phi_{\bar{z}}\right)\left[\left(B_{v}-B_{\bar{v}}\right)-\frac{B}{2}\left(\phi_{v}-\phi_{\bar{v}}\right)\right]\right]
$$$$
-e^{2356}\left[\frac{\left(\phi_{v}-\phi_{\bar{v}}\right)}{A}\left[\left(B_{z}-B_{\bar{z}}\right)-\frac{B}{2}\left(\phi_{z}-\phi_{\bar{z}}\right)\right]-e^{-a}\left(\phi_{z}+\phi_{\bar{z}}\right)\left[\left(B_{v}+B_{\bar{v}}\right)-\frac{B}{2}\left(\phi_{v}+\phi_{\bar{v}}\right)\right]\right]
$$$$
-i e^{2456}\left[\frac{\left(\phi_{v}+\phi_{\bar{v}}\right)}{A}\left[\left(B_{z}-B_{\bar{z}}\right)-\frac{B}{2}\left(\phi_{z}-\phi_{\bar{z}}\right)\right]-e^{-a}\left(\phi_{z}+\phi_{\bar{z}}\right)\left[\left(B_{v}-B_{\bar{v}}\right)-\frac{B}{2}\left(\phi_{v}-\phi_{\bar{v}}\right)\right]\right]
$$$$
+B\left[-i e^{-a}\left(e^{1236}+e^{1245}\right)\left(\phi_{z}-\phi_{\bar{z}}\right)-e^{-a}\left(e^{1246}-e^{1235}\right)\left(\phi_{z}+\phi_{\bar{z}}\right)\right.
$$$$
\left.\left.+i \frac{\left(\phi_{v}-\phi_{\bar{v}}\right)}{A}\left(e^{1346}+e^{2345}\right)+\frac{\left(\phi_{v}+\phi_{\bar{v}}\right)}{A}\left(e^{2346}-e^{1345}\right)\right]\right]
$$

$d W$ can be calculated as follows:

$$
d W=\frac{3}{4}\left[\dot{a}\left[i\left(\phi_{z}-\phi_{\bar{z}}\right) e^{-a} e^{1 t}-\left(\phi_{z}+\phi_{\bar{z}}\right) e^{-a} e^{2 t}\right]\right.
$$




$$
\begin{aligned}
& \left.+\left[e^{1 t} i\left(\dot{\phi}_{z}-\dot{\phi}_{\bar{z}}\right) e^{-a}+e^{2 t}\left(\dot{\phi}_{z}+\dot{\phi}_{\bar{z}}\right) e^{-a}+e^{3 t} i \frac{\left(\dot{\phi}_{v}-\dot{\phi}_{\bar{v}}\right)}{A}-e^{4 t} \frac{\left(\dot{\phi}_{v}+\dot{\phi}_{\bar{v}}\right)}{A}\right]-4 e^{12} \phi_{\bar{z} z} e^{-a}\right] \\
& -i e^{13}\left[\left(\phi_{z v}-\phi_{\bar{z} v}+\phi_{z \bar{v}}+\phi_{\bar{z} \bar{v}}\right) e^{-a}-\left(\phi_{v z}-\phi_{\bar{v} z}+\phi_{v \bar{z}}-\phi_{\bar{v} \bar{z}}\right) \frac{1}{A}+\frac{\left(A_{z}+A_{\bar{z}}\right)}{A^{2}}\left(\phi_{v}-\phi_{\bar{v}}\right)\right] \\
& +e^{14}\left[\left(\phi_{z v}-\phi_{\bar{z} v}-\phi_{z \bar{v}}+\phi_{\bar{z} \bar{v}}\right) e^{-a}-\left(\phi_{v z}+\phi_{\bar{v} z}+\phi_{v \bar{z}}+\phi_{\bar{v} \bar{z}}\right) \frac{1}{A}+\frac{\left(A_{z}+A_{\bar{z}}\right)}{A^{2}}\left(\phi_{v}+\phi_{\bar{v}}\right)\right] \\
& +e^{23}\left[\left(\phi_{z v}+\phi_{\bar{z} v}+\phi_{z \bar{v}}+\phi_{\bar{z} \bar{v}}\right) e^{-a}-\left(\phi_{v z}-\phi_{\bar{v} z}-\phi_{v \bar{z}}-\phi_{\bar{v} \bar{z}}\right) \frac{1}{A}+\frac{\left(A_{z}-A_{\bar{z}}\right)}{A^{2}}\left(\phi_{v}-\phi_{\bar{v}}\right)\right] \\
& +i e^{24}\left[\left(\phi_{z v}+\phi_{\bar{z} v}-\phi_{z \bar{v}}-\phi_{\bar{z} \bar{v}}\right) e^{-a}-\left(\phi_{v z}+\phi_{\bar{v} z}-\phi_{v \bar{z}}-\phi_{\bar{v} \bar{z}}\right) \frac{1}{A}+\frac{\left(A_{z}-A_{\bar{z}}\right)}{A^{2}}\left(\phi_{v}+\phi_{\bar{v}}\right)\right] \\
& +e^{34}\left[-4 \phi_{\bar{v} v}+\frac{2 A_{v} \phi_{\bar{v}}+2 A_{\bar{z}} \phi_{v}}{A^{2}}\right]
\end{aligned}
$$

$$
\begin{aligned}
& v \wedge J \wedge d W \\
& =\frac{3}{4} e^{\phi} d t \wedge\left[B\left[-e^{1256} 4 \phi_{\bar{z} z} e^{-a}\right]\right. \\
& -i e^{1356}\left[\left(\phi_{z v}-\phi_{\bar{z} v}+\phi_{z \bar{v}}+\phi_{\bar{z} \bar{v}}\right) e^{-a}-\left(\phi_{v z}-\phi_{\bar{v} z}+\phi_{v \bar{z}}-\phi_{\bar{v} \bar{z}}\right) \frac{1}{A}+\frac{\left(A_{z}+A_{\bar{z}}\right)}{A^{2}}\left(\phi_{v}-\phi_{\bar{v}}\right)\right] \\
& +e^{1456}\left[\left(\phi_{z v}-\phi_{\bar{z} v}-\phi_{z \bar{v}}+\phi_{\bar{z} \bar{v}}\right) e^{-a}-\left(\phi_{v z}+\phi_{\bar{v} z}+\phi_{v \bar{z}}+\phi_{\bar{v} \bar{z}}\right) \frac{1}{A}+\frac{\left(A_{z}+A_{\bar{z}}\right)}{A^{2}}\left(\phi_{v}+\phi_{\bar{v}}\right)\right] \\
& +e^{2356}\left[\left(\phi_{z v}+\phi_{\bar{z} v}+\phi_{z \bar{v}}+\phi_{\bar{z} \bar{v}}\right) e^{-a}-\left(\phi_{v z}-\phi_{\bar{v} z}-\phi_{v \bar{z}}-\phi_{\bar{v} \bar{z}}\right) \frac{1}{A}+\frac{\left(A_{z}-A_{\bar{z}}\right)}{A^{2}}\left(\phi_{v}-\phi_{\bar{v}}\right)\right] \\
& +i e^{2456}\left[\left(\phi_{z v}+\phi_{\bar{z} v}-\phi_{z \bar{v}}-\phi_{\bar{z} \bar{v}}\right) e^{-a}-\left(\phi_{v z}+\phi_{\bar{v} z}-\phi_{v \bar{z}}-\phi_{\bar{v} \bar{z}}\right) \frac{1}{A}+\frac{\left(A_{z}-A_{\bar{z}}\right.}{A^{2}}\left(\phi_{v}+\phi_{\bar{v}}\right)\right] \\
& +e^{3456}\left[-4 \phi_{\bar{v} v}+\frac{2 A_{v} \phi_{\bar{v}}+2 A_{\bar{z}} \phi_{v}}{A^{2}}\right] \\
& e^{1234}\left[-A 4 \phi_{\bar{z} z} e^{-a}+e^{a}\left[-4 \phi_{\bar{v} v}+\frac{\left.\left.2 A_{v} \phi_{\bar{v}}+2 A_{\bar{z}} \phi_{v}\right]\right]}{A^{2}}\right.\right. \\
& \quad d \star_{\star_{7}} S=-\frac{1}{2} d t \wedge\left[e^{1256}\left[2\left(A_{z z}+A_{\bar{z} \bar{z}}\right)-A\left(\phi_{z z}+\phi_{\bar{z} \bar{z}}\right)-A_{z} \phi_{z}-A_{\bar{z}} \phi_{\bar{z}}\right]\right. \\
& \quad-e^{2356}\left[A_{z v}+A_{\bar{z} \bar{v}}+A_{z \bar{v}}+A_{\bar{z} \bar{v}}-\frac{A}{2}\left(\phi_{z v}+\phi_{\bar{z} v}+\phi_{z \bar{v}}+\phi_{\bar{z} \bar{v}}\right)\right. \\
& \quad-\frac{\left(A_{v}+A_{\bar{v}}\right)}{2}\left(\phi_{z}+\phi_{\bar{z}}\right)+\frac{e^{a}}{2}\left(\phi_{v z}-\phi_{\bar{v} z}-\phi_{v \bar{z}}+\phi_{\bar{v} \bar{z})]}\right. \\
& \quad-i e^{2456}\left[A_{z v}+A_{\bar{z} \bar{v}}-A_{z \bar{v}}-A_{\bar{z} \bar{v}}-\frac{A}{2}\left(\phi_{z v}+\phi_{\bar{z} v}-\phi_{z \bar{v}}-\phi_{\bar{z} \bar{v}}\right)\right. \\
& \left.\quad\left(\phi_{z}+\phi_{\bar{z}}\right)+\frac{e^{a}}{2}\left(\phi_{v z}+\phi_{\bar{v} z}-\phi_{v \bar{z}}-\phi_{\bar{v} \bar{z}}\right)\right]
\end{aligned}
$$




$$
\begin{aligned}
& -i e^{1356}\left[A_{z v}-A_{\bar{z} \bar{v}}+A_{z \bar{v}}-A_{\bar{z} \bar{v}}-\frac{A}{2}\left(\phi_{z v}-\phi_{\bar{z} v}+\phi_{z \bar{v}}-\phi_{\bar{z} \bar{v}}\right)\right. \\
& \left.-\frac{\left(A_{v}+A_{\bar{v}}\right)}{2}\left(\phi_{z}-\phi_{\bar{z}}\right)+\frac{e^{a}}{2}\left(\phi_{v z}-\phi_{\bar{v} z}+\phi_{v \bar{z}}-\phi_{\bar{v} \bar{z}}\right)\right] \\
& +e^{1456}\left[A_{z v}-A_{\bar{z} \bar{v}}-A_{z \bar{v}}+A_{\bar{z} \bar{v}}-\frac{A}{2}\left(\phi_{z v}-\phi_{\bar{z} v}-\phi_{z \bar{v}}+\phi_{\bar{z} \bar{v}}\right)\right. \\
& \left.-\frac{\left(A_{v}-A_{\bar{v}}\right)}{2}\left(\phi_{z}-\phi_{\bar{z}}\right)+\frac{e^{a}}{2}\left(\phi_{v z}+\phi_{\bar{v} z}+\phi_{v \bar{z}}+\phi_{\bar{v} \bar{z}}\right)\right] \\
& +e^{1234}\left[2\left(B_{z z}+B_{\bar{z} \bar{z}}+B_{v v}+B_{\bar{v} \bar{v}}\right)-B\left(\phi_{z z}+\phi_{\bar{z} \bar{z}}+\phi_{v v}+\phi_{\bar{v} \bar{v}}\right)-B_{z} \phi_{z}-B_{\bar{z}} \phi_{\bar{z}}\right. \\
& \left.-B_{v} \phi_{v}-B_{\bar{v}} \phi_{\bar{v}}+4 B\right]-e^{3456} e^{a}\left(\phi_{v v}+\phi_{\bar{v} \bar{v}}\right) \\
& +\left(e^{1235}-e^{1246}\right)\left[B_{z}+B_{\bar{z}}-A_{z}-A_{\bar{z}}-\frac{A}{2}\left(\phi_{z}+\phi_{\bar{z}}\right)\right] \\
& +i\left(e^{2345}+e^{1346}\right)\left[B_{v}-B_{\bar{v}}-\frac{e^{a}}{2}\left(\phi_{v}-\phi_{\bar{v}}\right)\right] \\
& -i\left(e^{1245}+e^{1236}\right)\left[B_{z}-B_{\bar{z}}+A_{z}-A_{\bar{z}}-\frac{A}{2}\left(\phi_{z}-\phi_{\bar{z}}\right)\right] \\
& \left.+\left(-e^{1345}+e^{2346}\right)\left[B_{v}+B_{\bar{v}}+\frac{e^{a}}{2}\left(\phi_{v}+\phi_{\bar{v}}\right)\right]\right]+
\end{aligned}
$$

From the above, one can calculate $*_{11} d G$ as follows:

$$
\begin{aligned}
\star_{11} d G= & e^{4 \Delta} d x^{0} \wedge d x^{1} \wedge d x^{2} \wedge d x^{3} \wedge \\
& {\left[\left(\ddot{a} \frac{e^{-\phi}}{2} e^{a} A+\frac{9}{8} e^{\phi}\left[A e^{-a}\left(4 \phi_{z} \phi_{\bar{z}}\right)+\frac{e^{a}}{A}\left(4 \phi_{v} \phi_{\bar{v}}\right)\right]\right.\right.} \\
- & \frac{3}{2} e^{\phi}\left[e^{-a}\left[-A_{z} \phi_{\bar{z}}-A_{\bar{z}} \phi_{z}+A \phi_{z} \phi_{\bar{z}}\right]+\frac{e^{a}}{A} \phi_{v} \phi_{\bar{v}}\right] \\
+ & \frac{3}{2} e^{\phi}\left[-A 2 \phi_{\bar{z} z} e^{-a}+e^{a}\left[-2 \phi_{\bar{v} v}+\frac{A_{v} \phi_{\bar{v}}+A_{\bar{z}} \phi_{v}}{A^{2}}\right]\right] \\
- & \frac{1}{2}\left[2\left(B_{z z}+B_{\bar{z} \bar{z}}+B_{v v}+B_{\bar{v} \bar{v}}\right)-B\left(\phi_{z z}+\phi_{\bar{z} \bar{z}}+\phi_{v v}+\phi_{\bar{v} \bar{v}}\right)-B_{z} \phi_{z}-B_{\bar{z}} \phi_{\bar{z}}\right. \\
& \left.\left.-B_{v} \phi_{v}-B_{\bar{v}} \phi_{\bar{v}}+4 B\right]-\frac{1}{2} \dot{a} \dot{\phi} e^{-\phi} e^{a} A\right) e^{56} \\
+ & \left(\ddot{a} \frac{e^{-\phi}}{2} e^{a} B+\frac{9}{8} e^{\phi} B e^{-a} 4 \phi_{z} \phi_{\bar{z}}-\frac{3}{2} e^{\phi} e^{-a}\left[-B_{z} \phi_{\bar{z}}-B_{\bar{z}} \phi_{z}+B \phi_{z} \phi_{\bar{z}}\right]\right. \\
+ & \left.3 e^{\phi} B\left[-\phi_{\bar{z} z} e^{-a}\right]-\frac{1}{2}\left[2\left(A_{z z}+A_{\bar{z} \bar{z}}\right)-A\left(\phi_{z z}+\phi_{\bar{z} \bar{z}}\right)-A_{z} \phi_{z}-A_{\bar{z}} \phi_{\bar{z}}\right]-\frac{1}{2} \dot{a} \dot{\phi} e^{-\phi^{a}} e^{a}\right) e^{34} \\
+ & \left(\ddot{a} \frac{e^{-\phi}}{2} A B+\frac{9}{8} e^{\phi} \frac{B}{A} 4 \phi_{v} \phi_{\bar{v}}\right]-\frac{3}{2} e^{\phi} \frac{1}{A}\left[-B_{v} \phi_{\bar{v}}-B_{\bar{v}} \phi_{v}+B \phi_{v} \phi_{\bar{v}}\right] \\
+ & \left.\frac{3}{2} e^{\phi}\left[-2 \phi_{\bar{v} v}+\frac{A_{v} \phi_{\bar{v}}+A_{\bar{z}} \phi_{v}}{A^{2}}\right]+\frac{1}{2} e^{a}\left(\phi_{v v}+\phi_{\bar{v} \bar{v}}\right)-\frac{1}{2} \dot{a} \dot{\phi} e^{-\phi} e^{a} A B\right) e^{12}
\end{aligned}
$$




$$
\begin{aligned}
& -\frac{\dot{a}}{2} e^{-\phi}\left[\left(\frac{\partial}{\partial z}+\frac{\partial}{\partial \bar{z}}\right) A B\right] e^{2 t}-\frac{\dot{a}}{2} e^{-\phi}\left[\left(\frac{\partial}{\partial z}-\frac{\partial}{\partial \bar{z}}\right) A B\right] i e^{1 t} \\
& +\frac{\dot{a}}{4} e^{-\phi} e^{a}\left(\phi_{v}+\phi_{\bar{v}}\right) B e^{4 t}+\frac{\dot{a}}{4} e^{-\phi} e^{a}\left(\phi_{v}-\phi_{\bar{v}}\right) B e^{3 t} \\
& +\left(-\frac{9}{8} e^{\phi} B\left[-\left(\phi_{z}-\phi_{\bar{z}}\right)\left(\phi_{v}+\phi_{\bar{v}}\right) e^{-a}+\frac{1}{A}\left(\phi_{z}+\phi_{\bar{z}}\right)\left(\phi_{v}-\phi_{\bar{v}}\right)\right]\right. \\
& -\frac{3}{4} e^{\phi}\left[\frac{\left(\phi_{v}-\phi_{\bar{v}}\right)}{A}\left[\left(B_{z}+B_{\bar{z}}\right)-\frac{B}{2}\left(\phi_{z}+\phi_{\bar{z}}\right)\right]\right. \\
& \left.-e^{-a}\left(\phi_{z}-\phi_{\bar{z}}\right)\left[\left(B_{v}+B_{\bar{v}}\right)-\frac{B}{2}\left(\phi_{v}+\phi_{\bar{v}}\right)\right]\right] \\
& -\frac{3}{4} e^{\phi}\left[\left(\phi_{z v}-\phi_{\bar{z} v}+\phi_{z \bar{v}}+\phi_{\bar{z} \bar{v}}\right) e^{-a}-\left(\phi_{v z}-\phi_{\bar{v} z}+\phi_{v \bar{z}}-\phi_{\bar{v} \bar{z}}\right) \frac{1}{A}\right. \\
& \left.+\frac{\left(A_{z}+A_{\bar{z}}\right)}{A^{2}}\left(\phi_{v}-\phi_{\bar{v}}\right)\right]+\frac{1}{2}\left[A_{z v}-A_{\bar{z} \bar{v}}+A_{z \bar{v}}-A_{\bar{z} \bar{v}}-\frac{A}{2}\left(\phi_{z v}-\phi_{\bar{z} v}+\phi_{z \bar{v}}-\phi_{\bar{z} \bar{v}}\right)\right. \\
& \left.\left.-\frac{\left(A_{v}+A_{\bar{v}}\right)}{2}\left(\phi_{z}-\phi_{\bar{z}}\right)+\frac{e^{a}}{2}\left(\phi_{v z}-\phi_{\bar{v} z}+\phi_{v \bar{z}}-\phi_{\bar{v} \bar{z}}\right)\right]\right) i e^{24} \\
& +\left(-\frac{9}{8} e^{\phi} B\left[\left(\phi_{z}-\phi_{\bar{z}}\right)\left(\phi_{v}-\phi_{\bar{v}}\right) e^{-a}-\frac{1}{A}\left(\phi_{z}+\phi_{\bar{z}}\right)\left(\phi_{v}+\phi_{\bar{v}}\right)\right]\right. \\
& +\frac{3}{4} e^{\phi}\left[\frac{\left(\phi_{v}+\phi_{\bar{v}}\right)}{A}\left[\left(B_{z}+B_{\bar{z}}\right)-\frac{B}{2}\left(\phi_{z}+\phi_{\bar{z}}\right)\right]\right. \\
& \left.-e^{-a}\left(\phi_{z}-\phi_{\bar{z}}\right)\left[\left(B_{v}-B_{\bar{v}}\right)-\frac{B}{2}\left(\phi_{v}-\phi_{\bar{v}}\right)\right]\right] \\
& +\frac{3}{4} e^{\phi}\left[\left(\phi_{z v}-\phi_{\bar{z} v}-\phi_{z \bar{v}}+\phi_{\bar{z} \bar{v}}\right) e^{-a}-\left(\phi_{v} z+\phi_{\bar{v} z}+\phi_{v \bar{z}}+\phi_{\bar{v} \bar{z}}\right) \frac{1}{A}\right. \\
& \left.+\frac{\left(A_{z}+A_{\bar{z}}\right)}{A^{2}}\left(\phi_{v}+\phi_{\bar{v}}\right)\right]-\frac{1}{2}\left[A_{z v}-A_{\bar{z} \bar{v}}-A_{z \bar{v}}+A_{\bar{z} \bar{v}}-\frac{A}{2}\left(\phi_{z v}-\phi_{\bar{z} v}-\phi_{z \bar{v}}+\phi_{\bar{z} \bar{v}}\right)\right. \\
& \left.\left.-\frac{\left(A_{v}-A_{\bar{v}}\right)}{2}\left(\phi_{z}-\phi_{\bar{z}}\right)+\frac{e^{a}}{2}\left(\phi_{v z}+\phi_{\bar{v} z}+\phi_{v \bar{z}}+\phi_{\bar{v} \bar{z}}\right)\right]\right) e^{23} \\
& +\left(-\frac{9}{8} e^{\phi} B\left[\left(\phi_{z}+\phi_{\bar{z}}\right)\left(\phi_{v}+\phi_{\bar{v}}\right) e^{-a}-\frac{1}{A}\left(\phi_{z}-\phi_{\bar{z}}\right)\left(\phi_{v}-\phi_{\bar{v}}\right)\right]\right. \\
& +\frac{3}{4} e^{\phi}\left[\frac{\left(\phi_{v}-\phi_{\bar{v}}\right)}{A}\left[\left(B_{z}-B_{\bar{z}}\right)-\frac{B}{2}\left(\phi_{z}-\phi_{\bar{z}}\right)\right]\right. \\
& \left.-e^{-a}\left(\phi_{z}+\phi_{\bar{z}}\right)\left[\left(B_{v}+B_{\bar{v}}\right)-\frac{B}{2}\left(\phi_{v}+\phi_{\bar{v}}\right)\right]\right] \\
& +\frac{3}{4} e^{\phi}\left[\left(\phi_{z v}+\phi_{\bar{z} v}+\phi_{z \bar{v}}+\phi_{\bar{z} \bar{v}}\right) e^{-a}-\left(\phi_{v} z-\phi_{\bar{v} z}-\phi_{v \bar{z}}-\phi_{\bar{v} \bar{z}}\right) \frac{1}{A}\right. \\
& \left.+\frac{\left(A_{z}-A_{\bar{z}}\right)}{A^{2}}\left(\phi_{v}-\phi_{\bar{v}}\right)\right]+\frac{1}{2}\left[A_{z v}+A_{\bar{z} \bar{v}}+A_{z \bar{v}}+A_{\bar{z} \bar{v}}-\frac{A}{2}\left(\phi_{z v}+\phi_{\bar{z} v}+\phi_{z \bar{v}}+\phi_{\bar{z} \bar{v}}\right)\right. \\
& \left.\left.-\frac{\left(A_{v}+A_{\bar{v}}\right)}{2}\left(\phi_{z}-\phi_{\bar{z}}\right)+\frac{e^{a}}{2}\left(\phi_{v z}-\phi_{\bar{v} z}-\phi_{v \bar{z}}+\phi_{\bar{v} \bar{z}}\right)\right]\right) e^{14}
\end{aligned}
$$




$$
\begin{aligned}
& +\left(-\frac{9}{8} e^{\phi} B\left[\left(\phi_{z}+\phi_{\bar{z}}\right)\left(\phi_{v}-\phi_{\bar{v}}\right) e^{-a}-\frac{1}{A}\left(\phi_{z}-\phi_{\bar{z}}\right)\left(\phi_{v}+\phi_{\bar{v}}\right)\right]\right. \\
& +\frac{3}{4} e^{\phi}\left[\frac{\left(\phi_{v}+\phi_{\bar{v}}\right)}{A}\left[\left(B_{z}-B_{\bar{z}}\right)-\frac{B}{2}\left(\phi_{z}-\phi_{\bar{z}}\right)\right]\right. \\
& \left.-e^{-a}\left(\phi_{z}+\phi_{\bar{z}}\right)\left[\left(B_{v}-B_{\bar{v}}\right)-\frac{B}{2}\left(\phi_{v}-\phi_{\bar{v}}\right)\right]\right] \\
& +\frac{3}{4} e^{\phi}\left[\left(\phi_{z v}+\phi_{\bar{z} v}-\phi_{z \bar{v}}-\phi_{\bar{z} \bar{v}}\right) e^{-a}-\left(\phi_{v} z+\phi_{\bar{v} z}-\phi_{v \bar{z}}-\phi_{\bar{v} \bar{z}}\right) \frac{1}{A}\right. \\
& \left.+\frac{\left(A_{z}-A_{\bar{z}}\right)}{A^{2}}\left(\phi_{v}+\phi_{\bar{v}}\right)\right]+\frac{1}{2}\left[A_{z v}+A_{\bar{z} \bar{v}}-A_{z \bar{v}}-A_{\bar{z} \bar{v}}-\frac{A}{2}\left(\phi_{z v}+\phi_{\bar{z} v}-\phi_{z \bar{v}}-\phi_{\bar{z} \bar{v}}\right)\right. \\
& \left.\left.-\frac{\left(A_{v}-A_{\bar{v}}\right)}{2}\left(\phi_{z}+\phi_{\bar{z}}\right)+\frac{e^{a}}{2}\left(\phi_{v z}+\phi_{\bar{v} z}-\phi_{v \bar{z}}-\phi_{\bar{v} \bar{z}}\right)\right]\right) i e^{13} \\
& +\left(\frac{3}{4} e^{\phi} e^{-a} B\left(\phi_{z}-\phi_{\bar{z}}\right)+\frac{1}{2}\left[B_{z}-B_{\bar{z}}+A_{z}-A_{\bar{z}}-\frac{A}{2}\left(\phi_{z}-\phi_{\bar{z}}\right)\right]\right) i\left(e^{45}+e^{36}\right) \\
& +\left(\frac{3}{4} e^{\phi} e^{-a} B\left(\phi_{z}+\phi_{\bar{z}}\right)+\frac{1}{2}\left[B_{z}+B_{\bar{z}}-A_{z}-A_{\bar{z}}+\frac{A}{2}\left(\phi_{z}+\phi_{\bar{z}}\right)\right]\right)\left(e^{35}-e^{46}\right) \\
& +\left(-\frac{3}{4} e^{\phi} \frac{\left(\phi_{v}-\phi_{\bar{v}}\right)}{A} B-\frac{1}{2}\left[B_{v}-B_{\bar{v}}-\frac{e^{a}}{2}\left(\phi_{v}-\phi_{\bar{v}}\right)\right]\right) i\left(e^{25}+e^{16}\right) \\
& \left.+\left(-\frac{3}{4} e^{\phi} \frac{\left(\phi_{v}+\phi_{\bar{v}}\right)}{A} B-\frac{1}{2}\left[B_{v}+B_{\bar{v}}+\frac{e^{a}}{2}\left(\phi_{v}+\phi_{\bar{v}}\right)\right]\right)\left(e^{15}-e^{26}\right)\right]
\end{aligned}
$$

This is relevant to the interpretation of the uplift as $M 5$-branes wrapped around supersymmetric two-cycles calibrated by a generalized calibration $J$, but generically, away from the standard complex structure point in the moduli space of almost complex structures. In the absence of $M 5$-branes, $*_{11} d G=0$ would lead to a system of coupled non-linear partial differential equations which will, if the solutions exists, be very difficult to solve.

\section{B The conformal immersion in $\mathrm{C}^{3}$}

In this appendix, we give an example of a Riemann surface obtained as a Weierstraß representation of conformal immersion of a surface in $\mathbf{C}^{3}$, using $\phi=x \equiv \operatorname{Re}(\mathrm{z})$ (the "linear dilaton" ansatz) and $A, B \in \mathbf{R}$. As the solutions we get will not involve periodic $A, B$, $\phi$, they are relevant only locally to the (non-compact) seven-folds of $S U(3)$-structure.

One needs to solve the following differential equation (use $\frac{\partial A, B}{\partial y}=0$ )

$$
\left(\frac{1}{4} \frac{d^{2}}{d x^{2}}-\frac{1}{2 x} \frac{d}{d x}+x^{2}\right) B=0 .
$$

The two solutions, $B_{1,2}$ are given by:

$$
B_{1}(x)=x^{\frac{3}{2}} J_{\frac{3}{4}}\left(x^{2}\right), B_{2}(x)=x^{\frac{3}{2}} J_{-\frac{3}{4}}\left(x^{2}\right) .
$$


Hence, the corresponding two values for $A$ would be:

$$
\begin{aligned}
& A_{1}(x)=\frac{1}{2 \sqrt{x}}\left(-2 x^{2} J_{-\frac{1}{4}}\left(x^{2}\right)-3 J_{\frac{3}{4}}\left(x^{2}\right)+2 x^{2} J_{\frac{7}{4}}\left(x^{2}\right)\right) \\
& A_{2}(x)=\frac{1}{2 \sqrt{x}}\left(-2 x^{2} J_{-\frac{7}{4}}\left(x^{2}\right)-3 J_{-\frac{3}{4}}\left(x^{2}\right)+2 x^{2} J_{\frac{1}{4}}\left(x^{2}\right)\right) .
\end{aligned}
$$

Then the Weierstraß representation for conformal immersion of a surface (三 Riemann surface) in $\mathbf{C}^{3}$ (See [2] and references therein) is given by defining the following coordinates $X^{i=1, \ldots, 6}(z, \bar{z})$, of the Rieman surface in $\mathbf{C}^{3}$ :

$$
\begin{aligned}
& X^{1}+i X^{2}=i \int_{\Gamma}\left(A_{1}^{2} d z^{\prime}-B_{1}^{2} d \bar{z}^{\prime}\right), \\
& X^{1}-i X^{2}=i \int_{\Gamma}\left(B_{1}^{2} d z^{\prime}-A_{1}^{2} d \bar{z}^{\prime}\right), \\
& X^{3}=-\int_{\Gamma}\left(A_{1} B_{1} d z^{\prime}+A_{1} B_{1} d \bar{z}^{\prime}\right), \\
& X^{4}+i X^{5}=i \int_{\Gamma}\left(A_{2}^{2} d z^{\prime}-B_{2}^{2} d \bar{z}^{\prime}\right), \\
& X^{4}-i X^{5}=i \int_{\Gamma}\left(B_{2}^{2} d z^{\prime}-A_{2}^{2} d \bar{z}^{\prime}\right), \\
& X^{6}=-\int_{\Gamma}\left(A_{2} B_{2} d z^{\prime}+A_{2} B_{2} d \bar{z}^{\prime}\right),
\end{aligned}
$$

where $\Gamma$ is a contour in $\mathbf{C}(z, \bar{z})$. The $\mathbf{C}^{3}$ coordinates for values of $B_{1,2}$ and $A_{1,2}$ as given in (B2) and (B3), for the path $\Gamma=\{(x, y) \mid x=y\}$, is given in appendix B. The induced metric on the Riemann surface is then given by:

$$
d s^{2}=\left(A_{1}^{2}+A_{2}^{2}+B_{1}^{2}+B_{2}^{2}\right)^{2}|d z|^{2} .
$$

The condition for a conformal immersion (implying $g_{z z}=g_{\bar{z} \bar{z}}=0$ ):

$$
\left(\frac{\partial X^{1}}{\partial z}\right)^{2}+\left(\frac{\partial X^{2}}{\partial z}\right)^{2}+\left(\frac{\partial X^{3}}{\partial z}\right)^{2}+\left(\frac{\partial X^{4}}{\partial z}\right)^{2}+\left(\frac{\partial X^{5}}{\partial z}\right)^{2}+\left(\frac{\partial X^{6}}{\partial z}\right)^{2}=0
$$

is a quadric in $\mathbf{C P}^{5}$ :

$$
w_{1}^{2}+w_{2}^{2}+w_{3}^{2}+w_{4}^{2}+w_{5}^{2}+w_{6}^{2}=0,
$$

$w_{i}$ being homogeneous coordinates on $\mathbf{C P}^{5}$.

Along the path $\Gamma:\left\{\left(x^{\prime}, y^{\prime}\right) \mid y^{\prime}=x^{\prime}, 0 \leq x^{\prime} \leq x \neq 0, x<1\right\}$, one sees that:

$$
X^{1}+i X^{2}=i I_{2}-I_{1}, X^{3}=-I_{3} ; X^{4}+i X^{5}=i I_{5}-I_{4}, X^{6}=-I_{6},
$$

where

$$
\begin{aligned}
& I_{1} \equiv \int_{\Gamma}\left(A_{1}^{2}+B_{1}^{2}\right), I_{2} \equiv \int_{\Gamma}\left(A_{1}^{2}-B_{1}^{2}\right), I_{3} \equiv \int_{\Gamma} A_{1} B_{1} \\
& I 4_{\equiv} \int_{\Gamma}\left(A_{1}^{2}+B_{1}^{2}\right), I_{5} \equiv \int_{\Gamma}\left(A_{1}^{2}-B_{1}^{2}\right), I_{6} \equiv \int_{\Gamma} A_{1} B_{1} .
\end{aligned}
$$


The integrals $I_{i}, i=1, \ldots, 6$ are given as:

$$
\begin{aligned}
& I_{1}=\frac{x^{4}\left(\left(J_{-\frac{1}{4}}\left(x^{2}\right)\right)^{2}-J_{-\frac{5}{4}}\left(x^{2}\right) J_{\frac{3}{4}}\left(x^{2}\right)\right)}{4}+\frac{x^{4}\left(\left(J_{\frac{3}{4}}\left(x^{2}\right)\right)^{2}-J_{-\frac{1}{4}}\left(x^{2}\right) J_{\frac{7}{4}}\left(x^{2}\right)\right)}{4} \\
& +\frac{x^{4}\left(\left(J_{\frac{7}{4}}\left(x^{2}\right)\right)^{2}-J_{\frac{3}{4}}\left(x^{2}\right) J_{\frac{11}{4}}\left(x^{2}\right)\right)}{4}+\frac{\left(x^{2}\right)^{\frac{3}{2}}{ }_{2} F_{3}\left(\left\{\frac{3}{4}, \frac{5}{4}\right\},\left\{\frac{3}{2}, \frac{7}{4}, \frac{7}{4}\right\},-x^{4}\right)}{\sqrt{2} \Gamma\left(\frac{3}{4}\right) \Gamma\left(\frac{7}{4}\right)}+ \\
& \frac{3\left(x^{2}\right)^{\frac{3}{2}}{ }_{2} F_{3}\left(\left\{\frac{3}{4}, \frac{5}{4}\right\},\left\{\frac{7}{4}, \frac{7}{4}, \frac{5}{2}\right\},-x^{4}\right)}{8 \sqrt{2} \Gamma\left(\frac{7}{4}\right)}-\frac{3\left(x^{2}\right)^{\frac{7}{2}}{ }_{2} F_{3}\left(\left\{\frac{7}{4}, \frac{9}{4}\right\},\left\{\frac{11}{4}, \frac{11}{4}, \frac{7}{2}\right\},-x^{4}\right)}{28 \sqrt{2} \Gamma\left(\frac{7}{4}\right) \Gamma\left(\frac{11}{4}\right)} \\
& -\frac{\left(x^{2}\right)^{\frac{7}{2}}\left(363{ }_{2} F_{3}\left(\left\{\frac{5}{4}, \frac{7}{4}\right\},\left\{\frac{5}{2}, \frac{11}{4}, \frac{11}{4}\right\},-x^{4}\right)-56 x^{4}{ }_{2} F_{3}\left(\left\{\frac{9}{4}, \frac{11}{4}\right\},\left\{\frac{7}{2}, \frac{15}{4}, \frac{15}{4}\right\},-x^{4}\right)\right)}{2541 \sqrt{2} \Gamma\left(\frac{3}{4}\right) \Gamma\left(\frac{11}{4}\right)} \\
& I_{2}=\frac{x^{4}\left(\left(J_{-\frac{1}{4}}\left(x^{2}\right)\right)^{2}-J_{-\frac{5}{4}}\left(x^{2}\right) J_{\frac{3}{4}}\left(x^{2}\right)\right)}{4}-\frac{x^{4}\left(\left(J_{\frac{3}{4}}\left(x^{2}\right)\right)^{2}-J_{-\frac{1}{4}}\left(x^{2}\right) J_{\frac{7}{4}}\left(x^{2}\right)\right)}{4} \\
& +\frac{x^{4}\left(\left(J_{\frac{7}{4}}\left(x^{2}\right)\right)^{2}-J_{\frac{3}{4}}\left(x^{2}\right) J_{\frac{11}{4}}\left(x^{2}\right)\right)}{4}+\frac{\left(x^{2}\right)^{\frac{3}{2}}{ }_{2} F_{3}\left(\left\{\frac{3}{4}, \frac{5}{4}\right\},\left\{\frac{3}{2}, \frac{7}{4}, \frac{7}{4}\right\},-x^{4}\right)}{\sqrt{2} \Gamma\left(\frac{3}{4}\right) \Gamma\left(\frac{7}{4}\right)}+ \\
& \frac{3\left(x^{2}\right)^{\frac{3}{2}}{ }_{2} F_{3}\left(\left\{\frac{3}{4}, \frac{5}{4}\right\},\left\{\frac{7}{4}, \frac{7}{4}, \frac{5}{2}\right\},-x^{4}\right)}{8 \sqrt{2}\left(\Gamma\left(\frac{7}{4}\right)\right)^{2}}-\frac{3\left(x^{2}\right)^{\frac{7}{2}}{ }_{2} F_{3}\left(\left\{\frac{7}{4}, \frac{9}{4}\right\},\left\{\frac{11}{4}, \frac{11}{4}, \frac{7}{2}\right\},-x^{4}\right)}{28 \sqrt{2} \Gamma\left(\frac{7}{4}\right) \Gamma\left(\frac{11}{4}\right)} \\
& -\frac{\left(x^{2}\right)^{\frac{7}{2}}\left(363{ }_{2} F_{3}\left(\left\{\frac{5}{4}, \frac{7}{4}\right\},\left\{\frac{5}{2}, \frac{11}{4}, \frac{11}{4}\right\},-x^{4}\right)-56 x^{4}{ }_{2} F_{3}\left(\left\{\frac{9}{4}, \frac{11}{4}\right\},\left\{\frac{7}{2}, \frac{15}{4}, \frac{15}{4}\right\},-x^{4}\right)\right)}{2541 \sqrt{2} \Gamma\left(\frac{3}{4}\right) \Gamma\left(\frac{11}{4}\right)}
\end{aligned}
$$

$$
\begin{aligned}
& I_{3}=\frac{x^{4}}{180 \sqrt{2} \Gamma\left(\frac{3}{4}\right) \Gamma\left(\frac{7}{4}\right)^{2} \Gamma\left(\frac{11}{4}\right)}\left[-36 \sqrt{x^{2}} \Gamma\left(\frac{7}{4}\right) \Gamma\left(\frac{11}{4}\right){ }_{2} F_{3}\left(\left\{\frac{5}{4}, \frac{5}{4}\right\},\left\{\frac{3}{2}, \frac{7}{4}, \frac{9}{4}\right\},-x^{4}\right)\right. \\
& \left.\left.+\Gamma\left(\frac{3}{4}\right)\left(-27 \Gamma\left(\frac{11}{4}\right){ }_{2} F_{3}\left(\left\{\frac{5}{4}, \frac{5}{4}\right\},\left\{\frac{7}{4}, \frac{9}{4}, \frac{5}{2}\right\},-x^{4}\right)+5 x^{4} \Gamma\left(\frac{7}{4}\right){ }_{2} F_{3}\left(\left\{\frac{9}{4}, \frac{9}{4}\right\},\left\{\frac{11}{4}, \frac{13}{4}, \frac{7}{2}\right\},-x^{4}\right)\right)\right)\right]
\end{aligned}
$$

$$
\begin{aligned}
& I_{4}=\frac{x^{4}\left(\left(J_{-\frac{7}{4}}\left(x^{2}\right)\right)^{2}-J_{-\frac{11}{4}}\left(x^{2}\right) J_{-\frac{3}{4}}\left(x^{2}\right)\right)}{4}+\frac{x^{4}\left(\left(J_{-\frac{3}{4}}\left(x^{2}\right)\right)^{2}-J_{-\frac{7}{4}}\left(x^{2}\right) J_{\frac{1}{4}}\left(x^{2}\right)\right)}{4}+ \\
& \frac{x^{4}\left(\left(J_{\frac{1}{4}}\left(x^{2}\right)\right)^{2}-J_{-\frac{3}{4}}\left(x^{2}\right) J_{\frac{5}{4}}\left(x^{2}\right)\right)}{4}-\frac{4 \sqrt{2}{ }_{2} F_{3}\left(\left\{-\frac{3}{4},-\left(\frac{1}{4}\right)\right\},\left\{-\frac{3}{2}, \frac{1}{4}, \frac{1}{4}\right\},-x^{4}\right)}{\left(x^{2}\right)^{\frac{3}{2}} \Gamma\left(-\frac{3}{4}\right), \Gamma\left(\frac{1}{4}\right)}
\end{aligned}
$$




$$
\begin{aligned}
& -\frac{3{ }_{2} F_{3}\left(\left\{-\left(\frac{3}{4}\right),-\left(\frac{1}{4}\right)\right\},\left\{-\left(\frac{1}{2}\right), \frac{1}{4}, \frac{1}{4}\right\},-x^{4}\right)}{\sqrt{2}\left(x^{2}\right)^{\frac{3}{2}}\left(\Gamma\left(\frac{1}{4}\right)^{2}\right.}-\frac{3 \sqrt{2} \sqrt{x^{2}}{ }_{2} F_{3}\left(\left\{\frac{1}{4}, \frac{3}{4}\right\},\left\{\frac{1}{2}, \frac{5}{4}, \frac{5}{4}\right\},-x^{4}\right)}{\Gamma\left(\frac{1}{4}\right) \Gamma\left(\frac{5}{4}\right)} \\
& -\frac{4 \sqrt{2} \sqrt{x^{2}}\left(75{ }_{2} F_{3}\left(\left\{-\frac{1}{4}, \frac{1}{4}\right\},\left\{-\left(\frac{1}{2}\right), \frac{5}{4}, \frac{5}{4}\right\},-x^{4}\right)+8 x^{4}{ }_{2} F_{3}\left(\left\{\frac{3}{4}, \frac{5}{4}\right\},\left\{\frac{1}{2}, \frac{9}{4}, \frac{9}{4}\right\},-x^{4}\right)\right)}{75 \Gamma\left(-\frac{3}{4}\right) \Gamma\left(\frac{5}{4}\right)}
\end{aligned}
$$

$$
\begin{aligned}
& I_{5}=\frac{x^{4}\left(\left(J_{-\frac{7}{4}}\left(x^{2}\right)\right)^{2}-J_{-\frac{11}{4}}\left(x^{2}\right) J_{-\frac{3}{4}}\left(x^{2}\right)\right)}{4}-\frac{x^{4}\left(\left(J_{-\frac{3}{4}}\left(x^{2}\right)\right)^{2}-J_{-\frac{7}{4}}\left(x^{2}\right) J_{\frac{1}{4}}\left(x^{2}\right)\right)}{4}+ \\
& \frac{x^{4}\left(\left(J_{\frac{1}{4}}\left(x^{2}\right)\right)^{2}-J_{-\frac{3}{4}}\left(x^{2}\right) J_{\frac{5}{4}}\left(x^{2}\right)\right)}{4}-\frac{4 \sqrt{2}{ }_{2} F_{3}\left(\left\{-\left(\frac{3}{4}\right),-\left(\frac{1}{4}\right)\right\},\left\{-\left(\frac{3}{2}\right), \frac{1}{4}, \frac{1}{4}\right\},-x^{4}\right)}{\left(x^{2}\right)^{\frac{3}{2}} \Gamma\left(-\left(\frac{3}{4}\right)\right) \Gamma\left(\frac{1}{4}\right)} \\
& -\frac{3{ }_{2} F_{3}\left(\left\{-\frac{3}{4},-\frac{1}{4}\right\},\left\{-\frac{1}{2}, \frac{1}{4}, \frac{1}{4}\right\},-x^{4}\right)}{\sqrt{2}\left(x^{2}\right)^{\frac{3}{2}} \Gamma\left(\frac{1}{4}\right)^{2}}-\frac{3 \sqrt{2} \sqrt{x^{2}}{ }_{2} F_{3}\left(\left\{\frac{1}{4}, \frac{3}{4}\right\},\left\{\frac{1}{2}, \frac{5}{4}, \frac{5}{4}\right\},-x^{4}\right)}{\Gamma\left(\frac{1}{4}\right) \Gamma\left(\frac{5}{4}\right)} \\
& -\frac{4 \sqrt{2} \sqrt{x^{2}}\left(7{ }_{2} F_{3}\left(\left\{-\frac{1}{4}, \frac{1}{4}\right\},\left\{-\left(\frac{1}{2}\right), \frac{5}{4}, \frac{5}{4}\right\},-x^{4}\right)+8 x^{4}{ }_{2} F_{3}\left(\left\{\frac{3}{4}, \frac{5}{4}\right\},\left\{\frac{1}{2}, \frac{9}{4}, \frac{9}{4}\right\},-x^{4}\right)\right)}{75 \Gamma\left(-\frac{3}{4}\right) \Gamma\left(\frac{5}{4}\right)}
\end{aligned}
$$

$$
\begin{gathered}
I_{6}=\frac{\sqrt{2}}{3 \sqrt{x^{2}} \Gamma\left(-\frac{3}{4}\right) \Gamma\left(\frac{1}{4}\right)^{2} \Gamma\left(\frac{5}{4}\right)}\left[12 \Gamma\left(\frac{1}{4}\right) \Gamma\left(\frac{5}{4}\right){ }_{2} F_{3}\left(\left\{-\frac{1}{4},-\frac{1}{4}\right\},\left\{-\frac{3}{2}, \frac{1}{4}, \frac{3}{4}\right\},-x^{4}\right)\right. \\
\left.\left.+\Gamma\left(-\frac{3}{4}\right)\left(9 \Gamma\left(\frac{5}{4}\right){ }_{2} F_{3}\left(\left\{-\frac{1}{4}\right),-\frac{1}{4}\right\},\left\{-\frac{1}{2}, \frac{1}{4}, \frac{3}{4}\right\},-x^{4}\right)+x^{4} \Gamma\left(\frac{1}{4}\right){ }_{2} F_{3}\left(\left\{\frac{3}{4}, \frac{3}{4}\right\},\left\{\frac{1}{2}, \frac{5}{4}, \frac{7}{4}\right\},-x^{4}\right)\right)\right] .
\end{gathered}
$$

We thus see that the immersion is severely non-linear in nature for the "linear dilaton background".

\section{References}

[1] G. Dall'Agata, N. Prezas, $N=1$ geometries for $M$-theory and type IIA strings with fluxes,Phys. Rev. D 69, 066004 (2004) [arXiv:hep-th/0311146].

[2] B. G.Konopelchenko and G. Landolfi, Generalized Weierstraß representation for surfaces in multidimensional Riemann spaces, Jour. Geom. and Phys., 29, 319 (1999) [arXiv;math.DG/9804144]. 
[3] S. Kachru, M. B. Schulz and S. Trivedi, Moduli stabilization from fluxes in a simple IIB orientifold, JHEP 0310, 007 (2003) [arXiv:hep-th/0201028]; S. Kachru, M. B. Schulz, P. K. Tripathy and S. P. Trivedi, New supersymmetric string compactifications, JHEP 03 (2003) [arXiv:hep-th/0211182].

[4] K. Becker, M. Becker, P. S. Green, K. Dasgupta, Compactifications of Heterotic Strings on Non-Kähler Complex Manifolds: I, JHEP 0304, 007 (2003) [arXiv:hep-th/0301161].

[5] S. Kachru, R. Kallosh, A. Linde and S. P. Trivedi, De-Sitter vacua in string theory, Phys. Rev. D 68, 046005 (2003) [arXiv:hep-th/0301240].

[6] J. M. Maldacena, The large $N$ limit of superconformal field theories and supergravity, Adv. Theor. Math. Phys. 2, 231 (1998) [Int. J. Theor. Phys. 38, 1113 (1999)] [arXiv:hepth/9711200].

[7] R. Gopakumar and C. Vafa, M-Theory and topological strings-I, II [arXiv:hepth/9809187,9812127]; On the gauge theory/geometry correspondence, Adv. Theor. Math. Phys. 3. 1415 (1999) [arXiv: hep-th/9811131].

[8] A. Strominger, Superstrings With Torsion, Nucl. Phys. B 274, 253 (1986).

[9] K. Becker, M. Becker, P. S. Green, K. Dasgupta, E. Sharpe, Compactifications of Heterotic Strings on Non-Kähler Complex Manifolds: II, Nucl. Phys. B 678, 19 (2004) [arXiv:hepth/0310058].

[10] K. Becker, K. Dasgupta, Heterotic Strings with Torsion, JHEP 0211, 006 (2002) [arXiv:hepth/0209077].

[11] G. Curio and A. Krause, Four-flux and warped heterotic M-theory compactifications, Nucl. Phys. B 602, 172 (2001) [arXiv:hep-th/0012152]; Enlarging the parameter space of heterotic M-theory compactifications, Nucl. Phys. B 693, 195 (2004) [arXiv:hep-th/0308202].

[12] L. Randall and R. Sundrum, A large mass hierarchy from a small extra dimension, Phys. Rev. Lett. 83, 3370 (1999) [arXIv:hep-ph/9905221].

[13] G. L. Cardoso, G. Curio, G. Dall'agata, D. Lüst, P. Manousselis and G. Zoupanos, NonKähler String Backgrounds and their Five Torsion Classes, Nucl. Phys. B 652, 5 (2003) [arXiv:hep-th/0211118].

[14] S. Chiossi and S. Salamon, The intrinsic torsion of $S U(3)$ and $G_{2}$ structures, in Differential Geometryt, Valencia 2001 (River Edge, NJ) 115, Wolrd Sci. Publishing, 2002 [arXiv:math.DG/0202282]. 
[15] S. Gurrieri, J. Louis, A. Micu and D. Waldram, Mirror Symmetry in Generalized Calabi-Yau Compactifications, Nucl. Phys. B 654, 61 (2003) [arXiv:hep-th/0211102].

[16] J. Gutowski, S. Ivanov and G. Papadopoulos, Deformations of generalized calibrations and compact non- Kähler manifolds with vanishing first Chern class, arXiv:math.dg/0205012.

[17] J. P. Gauntlett, D. Martelli and D. Waldram, Superstrings with intrinsic torsion, Phys. Rev. D 69, 086002 (2004) [arXiv:hep-th/0302158].

[18] A. Fayyazuddin and D. J. Smith, Localized intersections of M5-branes and four-dimensional superconformal field theories, JHEP 9904, 030 (1999) [arXiv:hep-th/9902210]; B. Brinne, A. Fayyazuddin, T. Z. Husain and D. J. Smith, $N=1$ M5-brane geometries, JHEP 0103, 052 (2001) [arXiv:hep-th/0012194].

[19] A. Misra, Flow equations for uplifting half-flat to Spin(7) manifolds, arXiv:hep-th/0507147. 